\title{
Hotspots and radio lobes of quasars
}

\author{
G. Swarup Radio Astronomy Centre, Tata Institute of Fundamental Research, \\ PO Box 8, Ootacamund 643 001, India and Huygen's Laboratorium, University \\ of Leiden, Leiden, The Netherlands \\ R. P. Sinha Systems Applied Science Corporation, Hyattesville, MD 20784, USA \\ K. Hilldrup National Radio Astronomy Observatory, ${ }^{\star}$ Charlottesville, West \\ Virginia, W.Va 24944, USA
}

Received 1983 October 13; in original form 1983 July 29

Summary. Observations are described of 31 quasars mapped with the VLA which provides a resolution of about $0.4 \operatorname{arcsec}$ at $4.87 \mathrm{GHz}$. The sources were selected from those lying near the upper envelope of the largest angular size (LAS) - redshift ( $z$ ) diagram for the $3 \mathrm{C}, 4 \mathrm{C}$ and $\mathrm{B} 2$ samples of quasars. The outer lobes of about a quarter of these quasars have no significant hotspots, which may imply that the lobes are not currently being supplied with energy. About 60 per cent of the sources have at least one compact hotspot with size $<4 \mathrm{kpc}$ transverse to the source axis, containing at least 20 per cent of the lobe flux density. For two-thirds of such hotspots, the magnetic field is perpendicular, within about $\pm 30^{\circ}$, to the source axis. For extended hotspots, the magnetic field tends to be circumferential. No marked dependence is found of the linear size of hotspots on $z$, LAS or radio luminosity, but the fraction of the flux density from the hotspots to that from the radio lobes is strongly correlated with radio luminosity.

On average, about 90 per cent of the flux density of the central radio component coincident with the optical quasar appears to come from a compact unresolved core, and the balance from extended features, a few kiloparsecs in size.

\section{Introduction}

Much observational effort has been devoted to investigating whether certain radio structural parameters of double radio quasars depend on redshift. Particularly, it is found that the upper envelope to the largest angular size (LAS) - redshift ( $z$ ) diagram follows the line LAS $\propto z^{-1}$

\footnotetext{
^ The National Radio Astronomy Observatory is operated by Associated Universities Inc., under contract with the National Science Foundation.
} 
(Miley 1971; Wardle \& Miley 1974). Correlations have also been reported between the angular sizes of the hotspots and redshift (Readhead \& Hewish 1976), and their compactness with radio luminosity (Jenkins \& McEllin 1977). It is conceivable, however, that selection and projection effects might influence the observed parameters whose dependence on redshift and on any other quasar characteristic is being investigated.

To minimize the potential confusion caused by such selection and projection effects, one may study complete samples of quasars located close to the upper envelope of the LAS $-z$ diagram. We expect these to be oriented at large angles to the line of sight. Such a choice also implies more detailed mapping for a given angular resolution. This paper describes observations of 31 such quasars made with the Very Large Array (VLA), which provides a resolution of about $0.4 \mathrm{arcsec}$ at a frequency of $4.87 \mathrm{GHz}$. This resolution is usually adequate to separate the hotspots from the surrounding diffuse emission of the outer radio lobes. Preliminary results of this study have been reported by Swarup, Sinha \& Salter (1982b). The observed sample and the observations are described in the next section. The following two sections outline the results obtained and a discussion of them.

\section{Observations and data reduction}

The selection criterion for the sample of 31 quasars described here is as follows: Firstly, from the 3CR and 4C complete samples (Hooley, Longair \& Riley 1978; Stannard \& Neal 1977; Wills 1979) we selected 28 quasars as being representative of all those lying near the upper envelope of the LAS $-z$ diagram. These quasars are distributed roughly uniformly over the seven successive redshift ranges of $<0.25,0.25-0.5,0.5-0.75,0.75-1,1-1.5,1.5-2$ and $>2$. Secondly, we selected all six quasars with $z>0.8$ from the B2 sample (Fanti et al. 1977, with redshifts taken from Hewitt \& Burbidge 1980), three of which are already present in our $4 \mathrm{C}$ sample. In order to minimize the scatter in radio luminosity of the selected quasars of similar angular size, preference was given to the $3 \mathrm{CR}$ sources at lower redshifts and to the $4 \mathrm{C}$ and $\mathrm{B} 2$ samples at higher redshifts. The LAS $-z$ distribution of the selected sample as well as of the complete $3 \mathrm{CR}, 4 \mathrm{C}$ and $\mathrm{B} 2$ samples is given in Section 6.

The observations were made with 22 antennas in the VLA 'A' configuration (Thompson et al. 1980) on 1981 March 17, 24 and 25 at $4873 \mathrm{MHz}$ using a bandwidth of $25 \mathrm{MHz}$. Each source was observed in two or three different scans of 4 or 5 min duration, separated by a few hours. To calibrate the amplitude and phases of the observed visibilities, one of 12 point sources was observed about every $15 \mathrm{~min}$.

The primary flux density calibration was made by observing 3C 286 whose flux density at $4.87 \mathrm{GHz}$ was taken as $7.41 \mathrm{Jy}$ on the scale of Baars et al. (1977). For polarization calibration, the radio sources 3C 138 and 3C 286 were observed. A uniform grading was applied across the aperture during the data reduction in order to obtain the highest possible resolution. Most of the sources were 'cleaned' (Högbom 1974) using the Westerbork software package at Leiden. Thirteen of the observed sources were self-calibrated (Schwab 1980). In order to estimate the flux density of the extended radio lobes, the dirty maps and the dirty beams of most of the observed sources were smoothed to a resolution of about $1-2$ arcsec, and then cleaned using the Groningen GIPSY software at Ootacamund. As shown in Table 1, the estimated flux densities of the radio lobes agree reasonably well with the measurements by other workers at $5 \mathrm{GHz}$, excepting a few sources which have relatively large diffuse lobes.

\section{Results}

\subsection{OBSERVATIONAL RESULTS}

The results for the 31 quasars are summarized in Table 1, which is arranged as follows: 


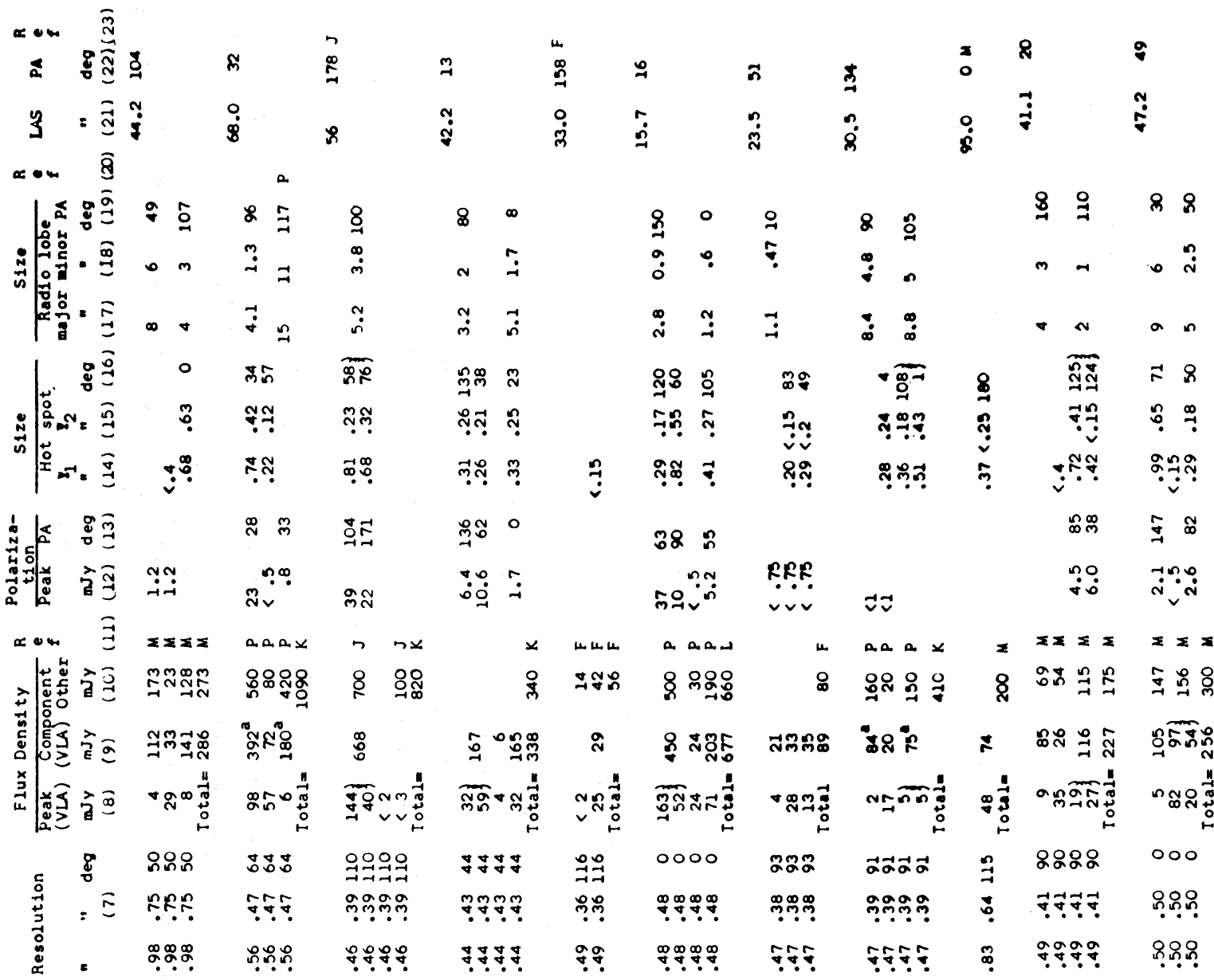

\begin{tabular}{|c|c|c|c|c|c|c|c|c|c|c|c|}
\hline out & $\bar{\sigma}=$ & s & n & 3 & $x u$ & $<$ & $\omega$ & is & $=$ & $x$ & $x$ \\
\hline 空 & 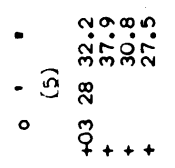 & 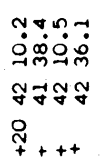 & 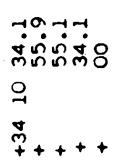 & 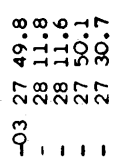 & 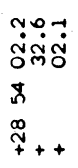 & 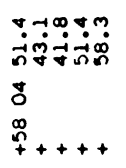 & 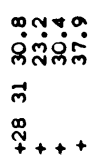 & 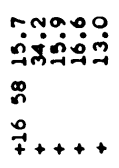 & 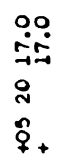 & 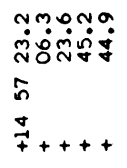 & 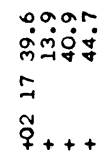 \\
\hline$x$ & 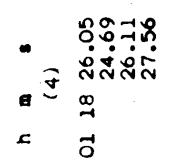 & 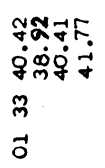 & 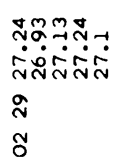 & 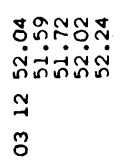 & 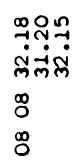 & 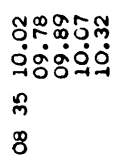 & 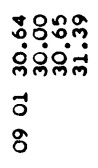 & 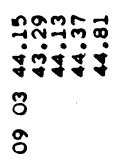 & 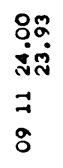 & 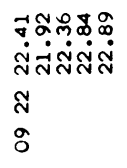 & 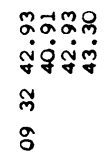 \\
\hline gे & 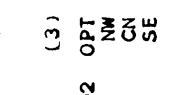 & 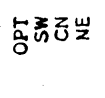 & 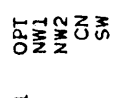 & 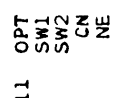 & 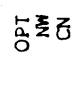 & 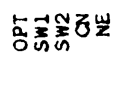 & 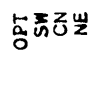 & 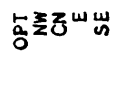 & 0 & 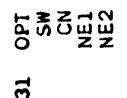 & $5=$ \\
\hline
\end{tabular}

\begin{tabular}{|c|c|c|c|c|c|c|c|}
\hline 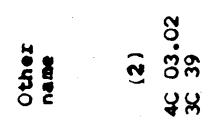 & $\dot{y}$ & $\begin{array}{l}\overrightarrow{\mathbf{\omega}} \\
\mathbf{0} \\
\dot{\phi}\end{array}$ & $\begin{array}{l}\overrightarrow{7} \\
\dot{0} \\
\stackrel{9}{y}\end{array}$ & జ & $\begin{array}{l}\text { N } \\
\text { u్ }\end{array}$ & & \\
\hline 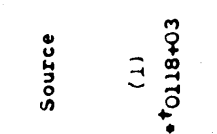 & 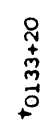 & 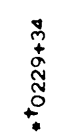 & 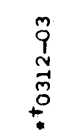 & 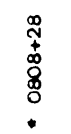 & 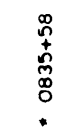 & & \\
\hline
\end{tabular}




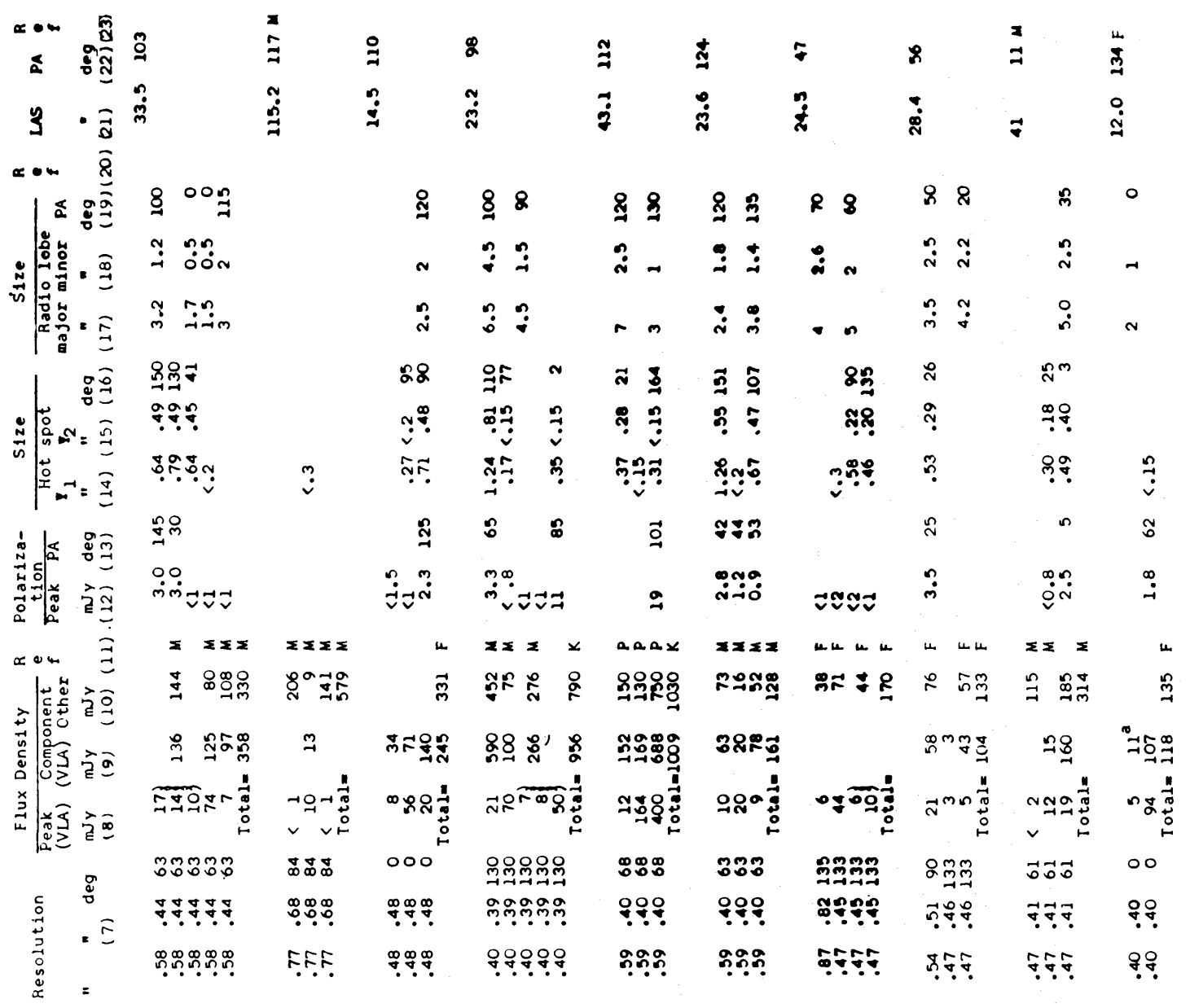

\begin{tabular}{|c|c|c|c|c|c|c|c|c|c|c|c|}
\hline & & $\Sigma$ & $z \Sigma \Sigma$ & z & a & a & $=$ & z & $z$ & $\leq \Sigma$ & D \\
\hline$x<+$ & $e$ & & roas & מ־ד. & 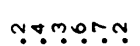 & ) & norm. & orm?\%? & mara & a.0. & 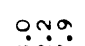 \\
\hline 5 & $=$ & 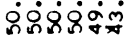 & $\dot{m} \dot{0} \dot{0} \dot{m}=\dot{二}$ & ஸ்்่ & ஜ்シ்ட்ல் & 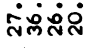 & piging & 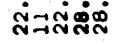 & 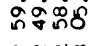 & 풍ำ & กู่ิ่ \\
\hline 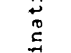 & $-\bar{n}$ & 9 & ஜะ๊๐ & กัญก๊ & $\cong$ & ¿ & m & $a$ & ఇ ఐల్ల్ల్ల & ติธก็ำ & $\vec{ल} \tilde{ल} \vec{m}$ \\
\hline & & $\wp_{+}$ & $\stackrel{m}{++++}$ & $\stackrel{\infty}{+}+++$ & $\stackrel{\tau}{+}$ & $\stackrel{\leftrightarrow}{+++}_{++}$ & $7+++$ & $\tilde{q}++++$ & $\stackrel{8}{++++}$ & ఫ̆+++ & $\overrightarrow{\tilde{+}}++$ \\
\hline & & 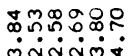 & 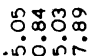 & 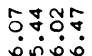 & 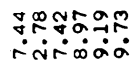 & 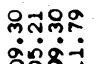 & 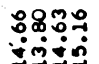 & 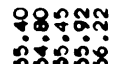 & 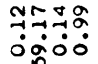 & 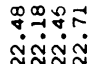 & 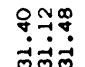 \\
\hline & " & 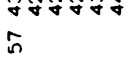 & $\begin{array}{l}d \\
2124\end{array}$ & $\begin{array}{l}\text { Jat } \\
8+2\end{array}$ & $8^{8 \text { กลกลก }}$ & and & $\vec{\lambda}$ & 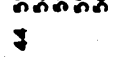 & 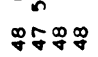 & $\begin{array}{l}8 \\
\text { NNAN }\end{array}$ & 孹m \\
\hline & & g & 9 & $\stackrel{9}{9}$ & $\exists$ & $\exists$ & 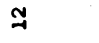 & $\mp$ & $\nexists$ & $\dddot{7}$ & 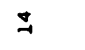 \\
\hline
\end{tabular}

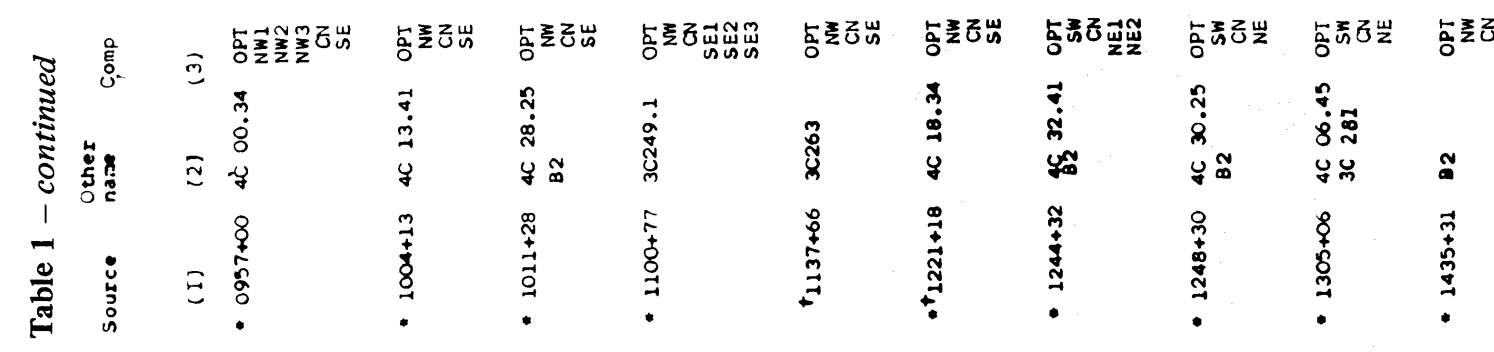




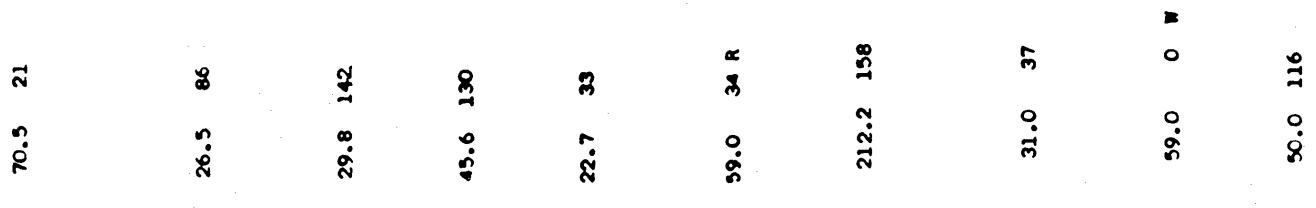

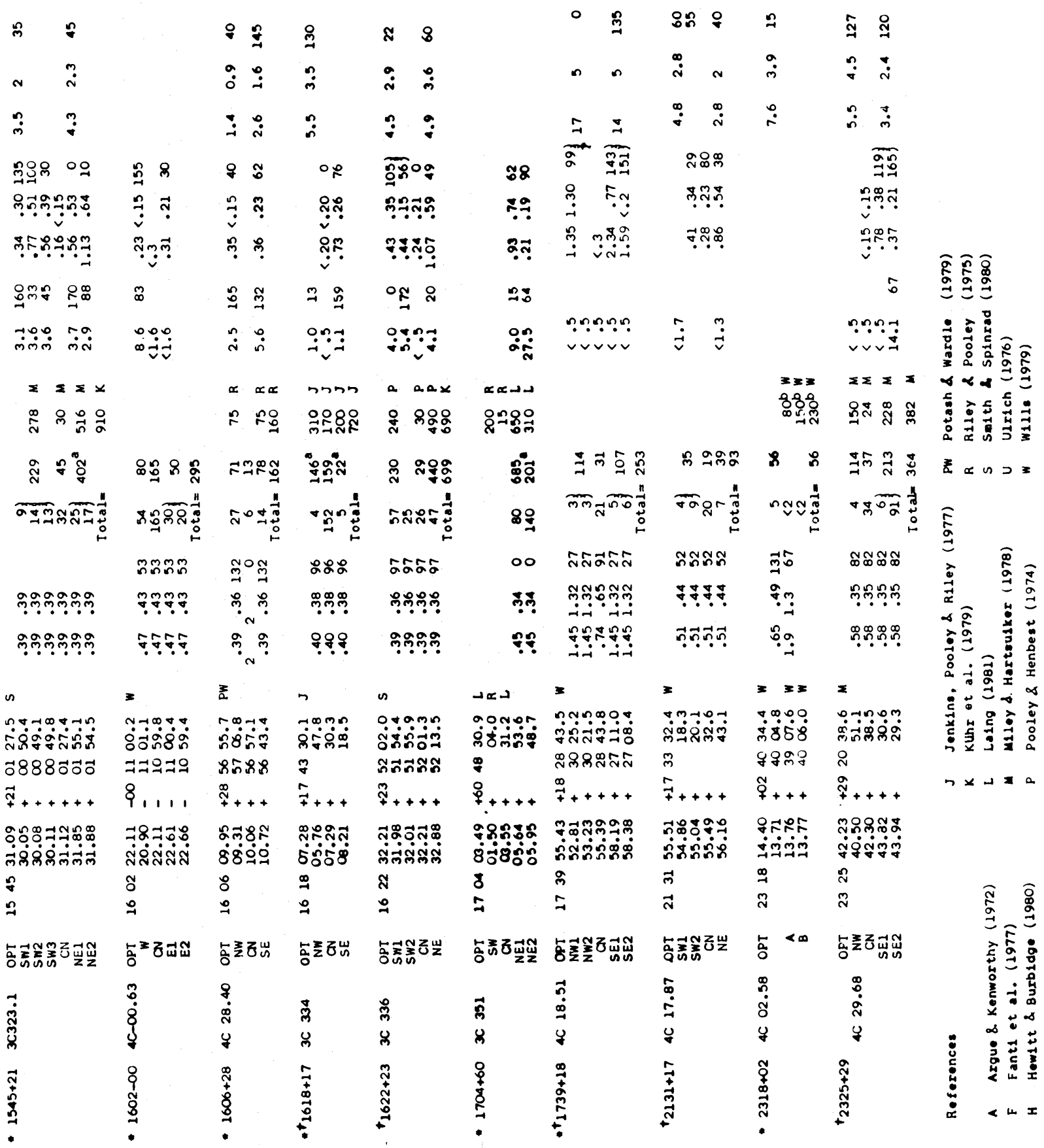


(1) Source name. An asterisk (*) before the source name indicates that a note is given in Section 3.3. A dagger $(\dagger)$ indicates that the map was self-calibrated.

(2) $3 \mathrm{C}, 4 \mathrm{C}$ or $\mathrm{B} 2$ name.

(3) Component designation.

(4) and (5) RA and Dec(1950.0). The optical positions are accurate to about \pm 0.5 arcsec. The radio positions are accurate to about \pm 0.1 arcsec for peak values of flux density $>10 \mathrm{mJy}$, although the errors are larger for smaller peak values.

(6) Reference for optical positions.

(7) Size of the antenna beam and its position angle.

(8) Peak values of flux density per beam area for the hotspots and for the core of the central components.

(9) Integrated flux density of each component obtained from the VLA maps, typically accurate to about 10 per cent. The superscript ' $a$ ' indicates that extended features are resolved out by the VLA and therefore the flux density is underestimated.

(10) and (11) Integrated flux density at $5 \mathrm{GHz}$ from the literature and reference. Superscript 'b' refers to values at $2.7 \mathrm{GHz}$ by Wills (1979).

(12) and (13) Peak value of the linearly polarized intensity and its position angle.

(14), (15) and (16) De-convolved full width to half-power (FWHP) of the hotspot or the central component along its major and minor axis in arcsec, and the position angle.

(17), (18) and (19) Approximate FWHP and position angle of radio lobes obtained from the low-resolution maps by removing the contribution of hotspots by eye estimates.

(20) Reference for FWHP of the radio lobes taken from the literature.

(21), (22) and (23) Largest angular size in arcsec, and position angle of the radio source, with reference when the values are from the literature.

\subsection{THE MAPS}

Fig. 1(i-xxviii) give contour plots of 28 quasars. No maps are given for the quasars $0808+$ $28,0911+05$ and $1004+13$ because the extended features are found to be resolved out fully at $4.87 \mathrm{GHz}$ by the VLA 'A'-configuration (see Section 3.3). The figure captions describe the maps.

\subsection{NOTES ON THE SOURCES}

$0118+03$. The source has a relaxed morphology with a weak hotspot in the SE component.

$0133+20$. The source has an ' $\mathrm{S}$ '-shaped geometry (Pooley \& Henbest 1974). The diffuse features of the source are resolved out by the VLA, particularly for the NE component. According to Pooley \& Henbest (1974), the peak feature in the NE component has a size $\sim 15 \mathrm{kpc}$.

$0229+34$. Only the north-west component of this source is seen in the VLA map. In addition there exists a very faint radio component $46 \operatorname{arcsec}$ to the south of the NW component (Mackay 1969). The quasar lies between these two components.

$0808+28$. The source has a strong central component coincident with the quasar and a weak diffuse NW component about 13 arcsec west and 30 arcsec north of the central component (Fanti et al. 1977). This latter component is resolved out by the VLA 'A' configuration. The flux density of the central component is found to be only $\sim 2 / 3$ of the value measured by Fanti et al. (1977), so either it is variable or it may have been partially resolved by the VLA beam. No other components are seen in the VLA maps obtained by smoothing 

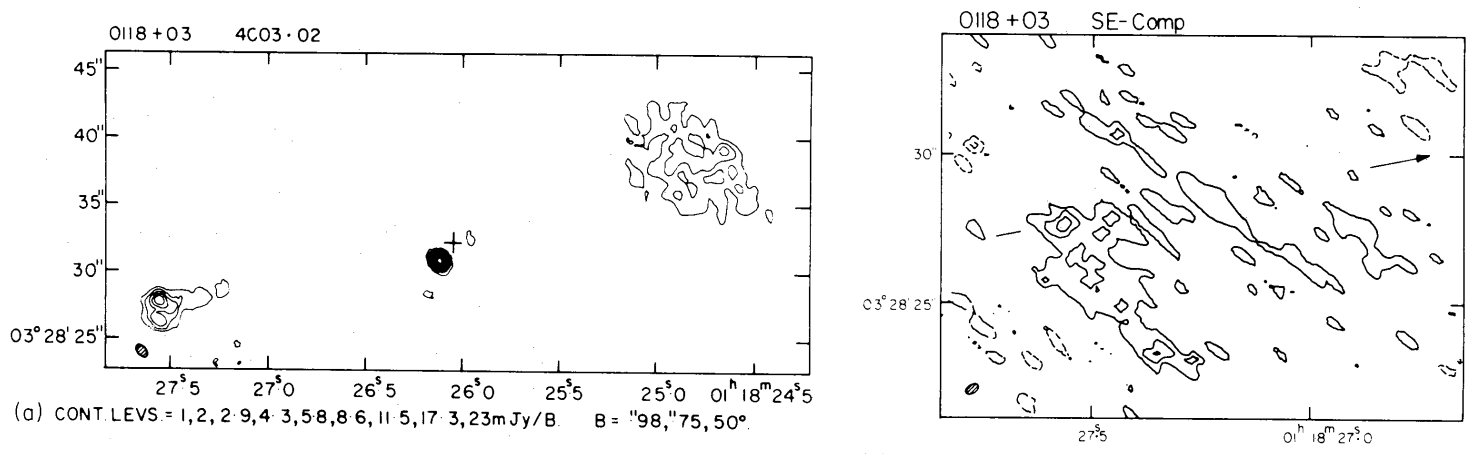

(b) CONT.LEVS. $=-2,-1,1,2,4 \mathrm{mJy} / 8 . \quad B=" 46, " 34,125^{\circ}$

(i)
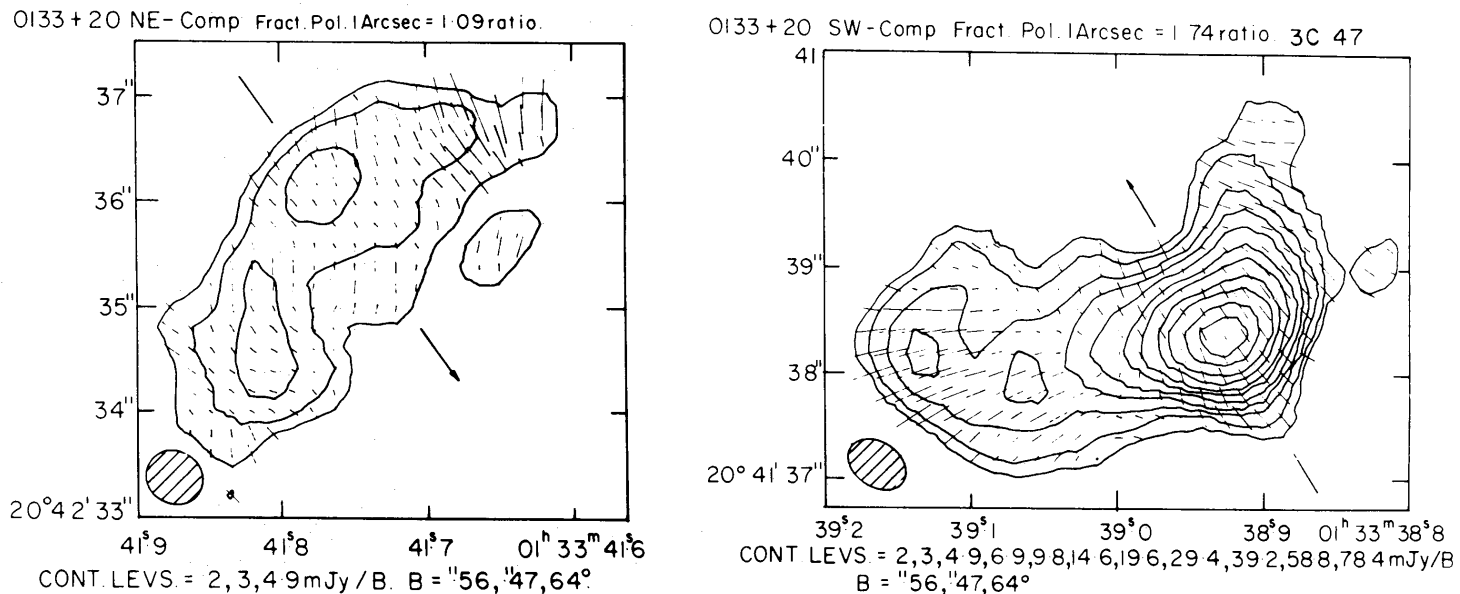

(ii)

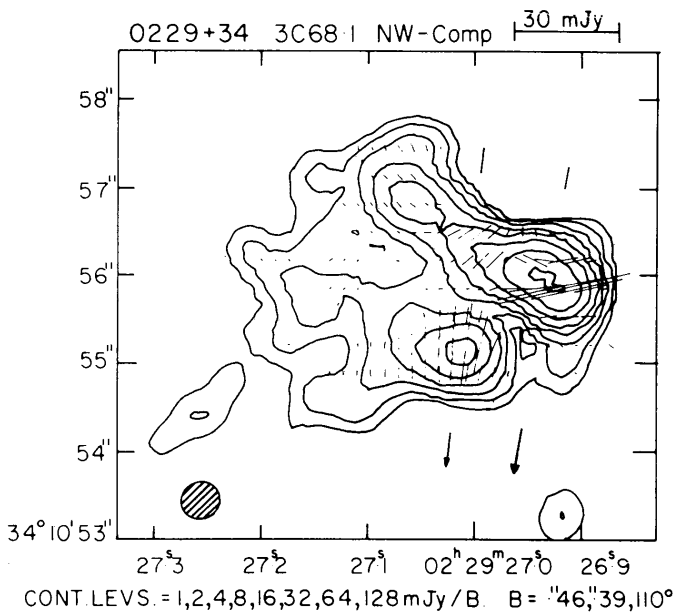

(iii)

Figure 1. Total-intensity contour maps of 28 quasars at $4.87 \mathrm{GHz}$. The broken lines indicate negative contours. The values of contour levels and the angular size of the VLA beam are given below each map, and the size of the beam is also indicated by a shaded ellipse. The superposed vectors represent the linearly polarized flux density, whose scale is given on the top right-hand side. In the maps of components, the arrows indicate the direction of the quasar nuclei, the optical positions of which are shown by crosses, if full maps are published. 
Figure 1 - continued

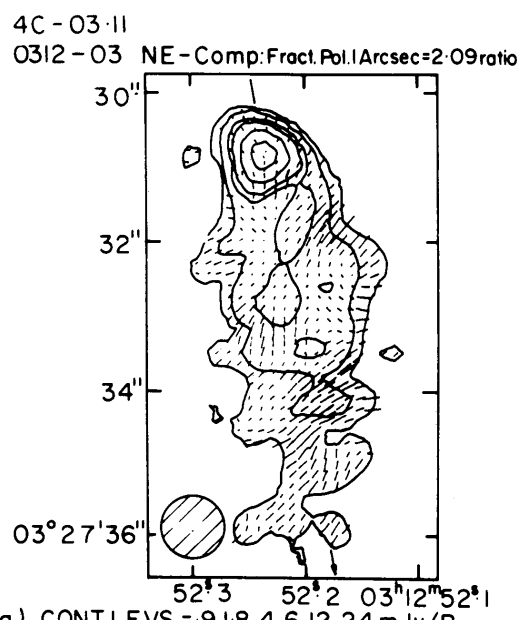

(a) CONT. LEVS $=9,1 \cdot 8,4,6,12,24 \mathrm{mJy} / \mathrm{B}$

$B=0.44,0.43,44^{\circ}$.

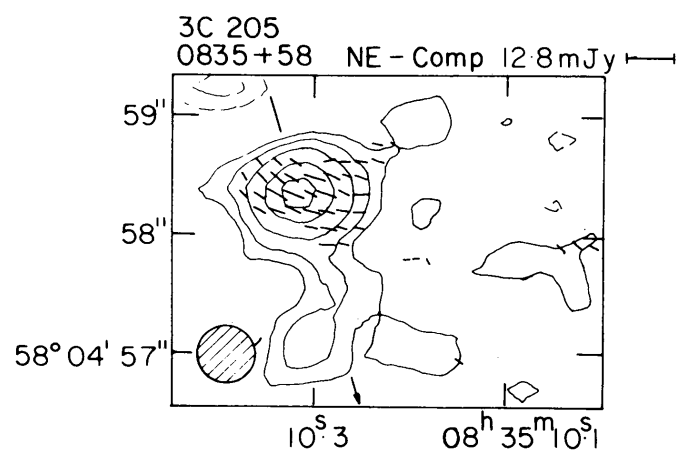

(a). CONT. LEVS. $=-10,-5,5,10,20,40,60,80 \mathrm{mJy} / \mathrm{B}$

$B=.48, " 48,0^{\circ}$.

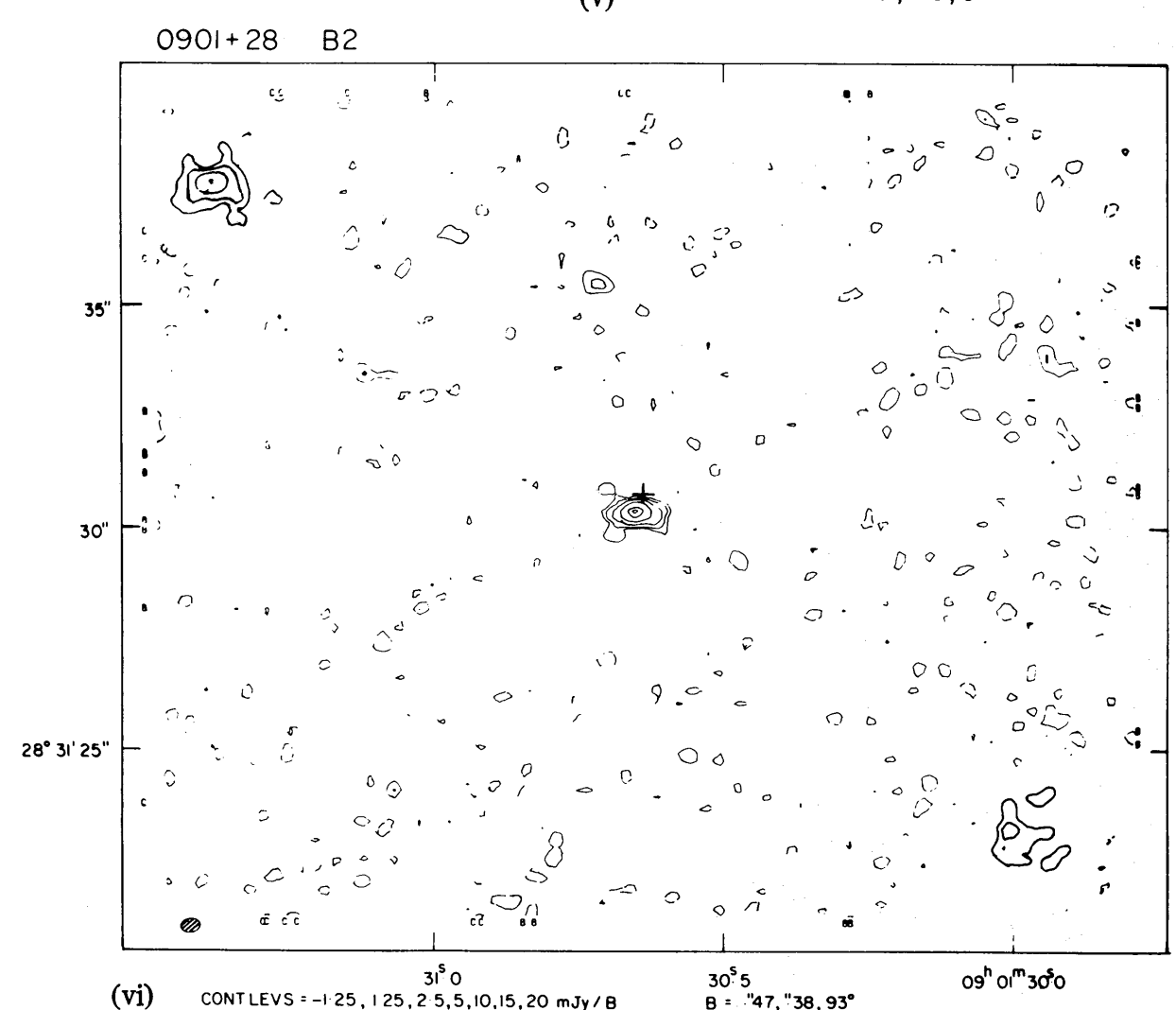

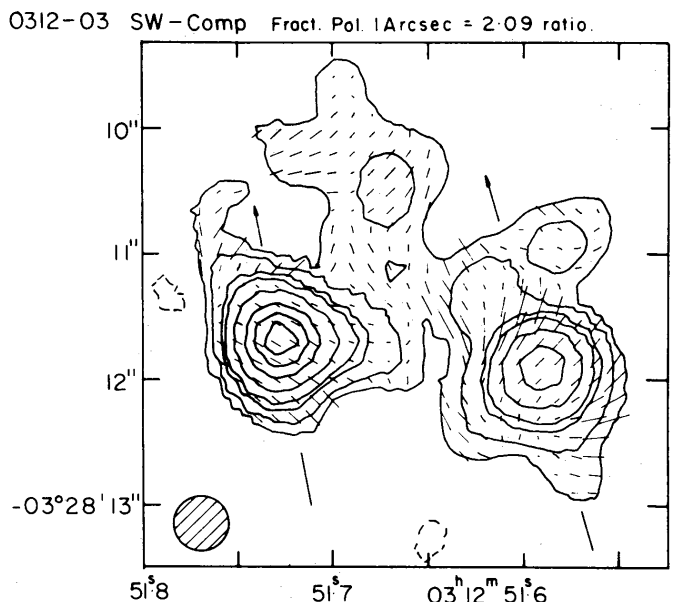

(iv)

b). CONT. LEVS $=-9,9,1 \cdot 8,4,6,12,24,35,47 \mathrm{~m} \mathrm{Jy} / \mathrm{B}$ $B=0.44,0.43,44^{\circ}$.

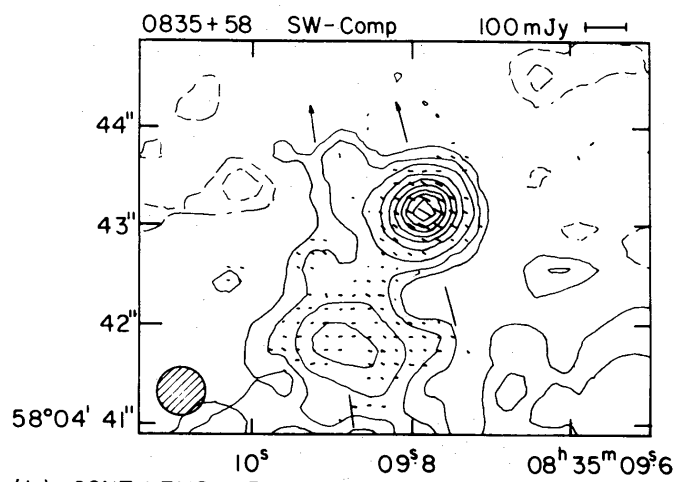

(b). CONT. LEVS. $=-5,-10,5,10,20,40,80,100,120,140 \mathrm{mJy} / \mathrm{B}$ $\mathrm{B}=.48, " 48,0^{\circ}$. 

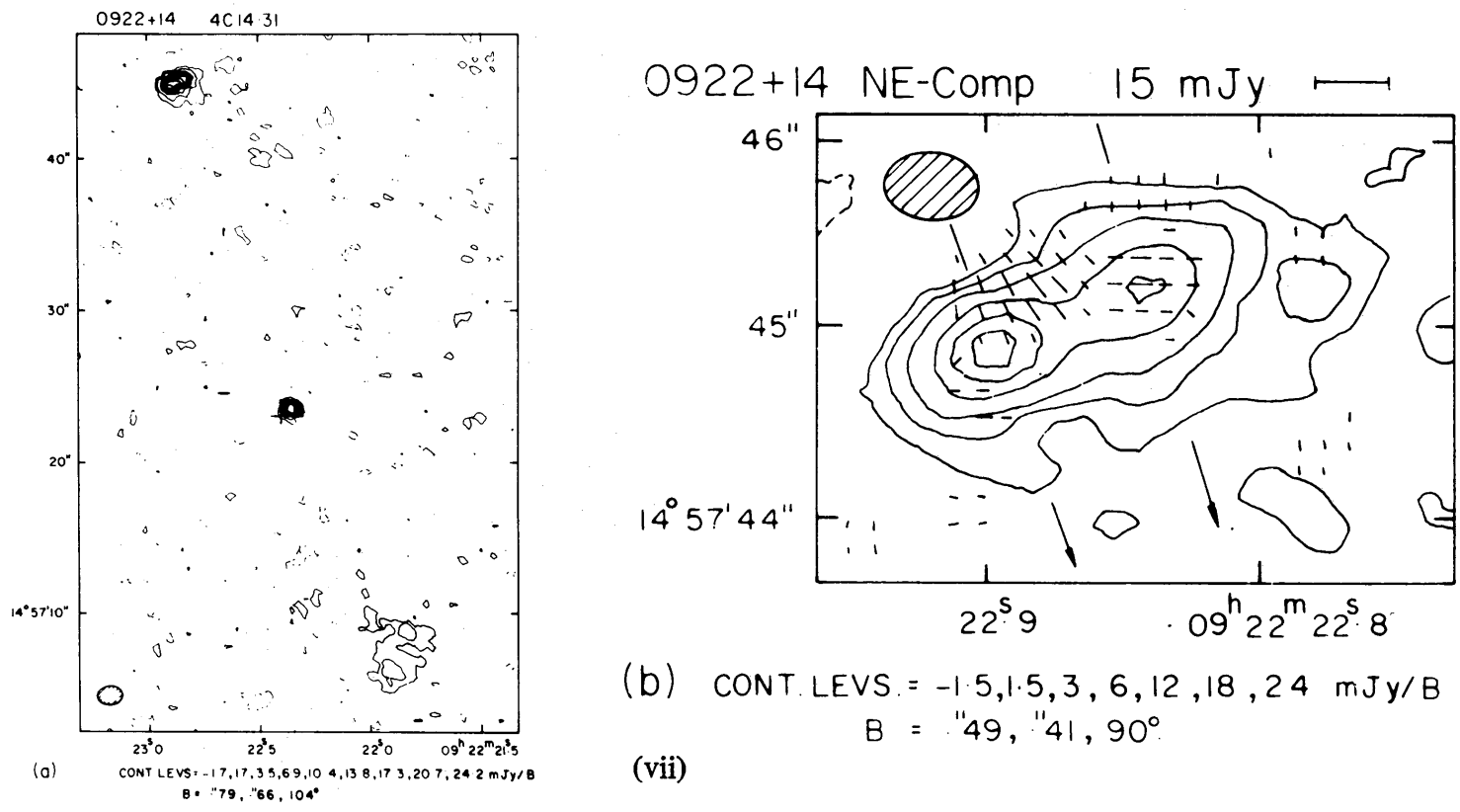

(b) CONT LEVS $=-1 \cdot 5,1 \cdot 5,3,6,12,18,24 \mathrm{mJy} / \mathrm{B}$

$B=49, .41,90^{\circ}$
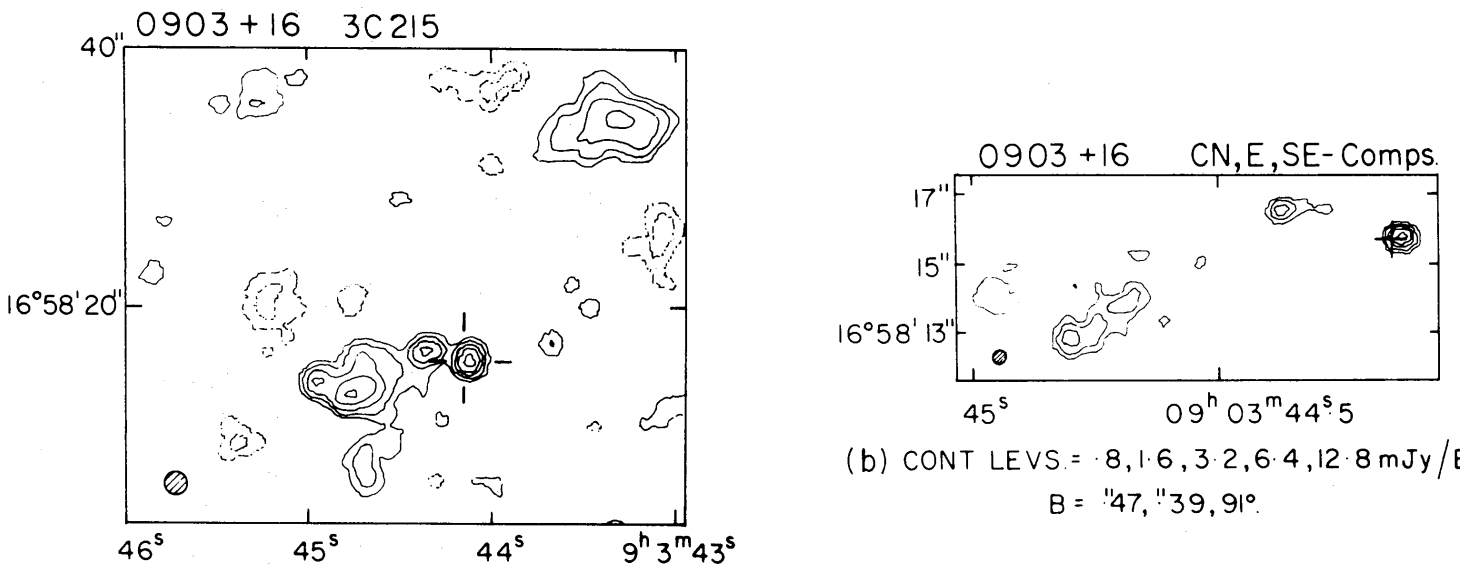

(b) CONT LEVS $=\cdot 8,1 \cdot 6,3 \cdot 2,6 \cdot 4,12 \cdot 8 \mathrm{mJy} / \mathrm{B}$

$B=" 47, " 39,91^{\circ}$.

(a) CONT. LEVS $=-1 \cdot 6,-8,8,8,1 \cdot 6,3 \cdot 2,6 \cdot 4,12 \cdot 8 \mathrm{mJy} / \mathrm{B}$

$B=1 " 51,1.38,116^{\circ}$.

(viii)

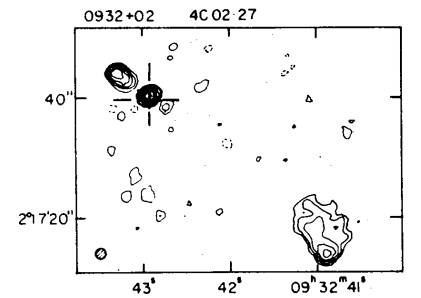

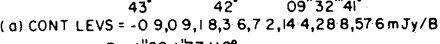
$B=1 " 60,1 " 37,119^{\circ}$

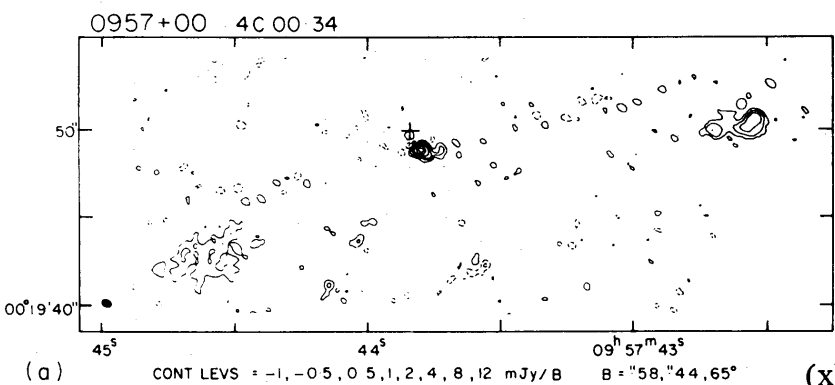

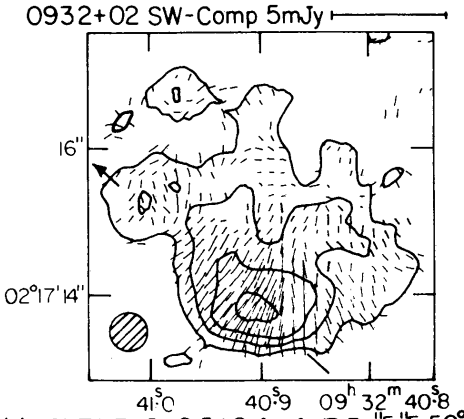

(ix) (c..CONT.LEVS. $=0 \cdot 5,1,2,4 \mathrm{~m}$ Jy/B. $B=" 5: 5,50^{\circ}$.

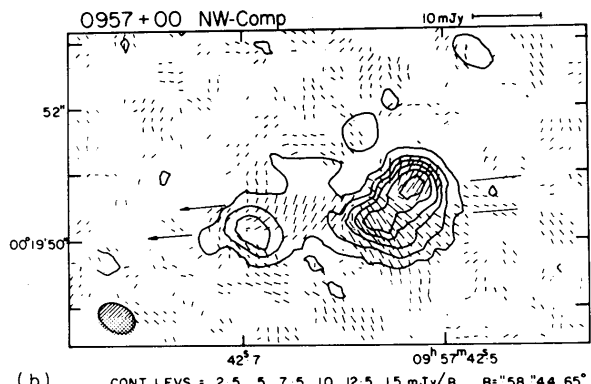


Figure 1 - continued

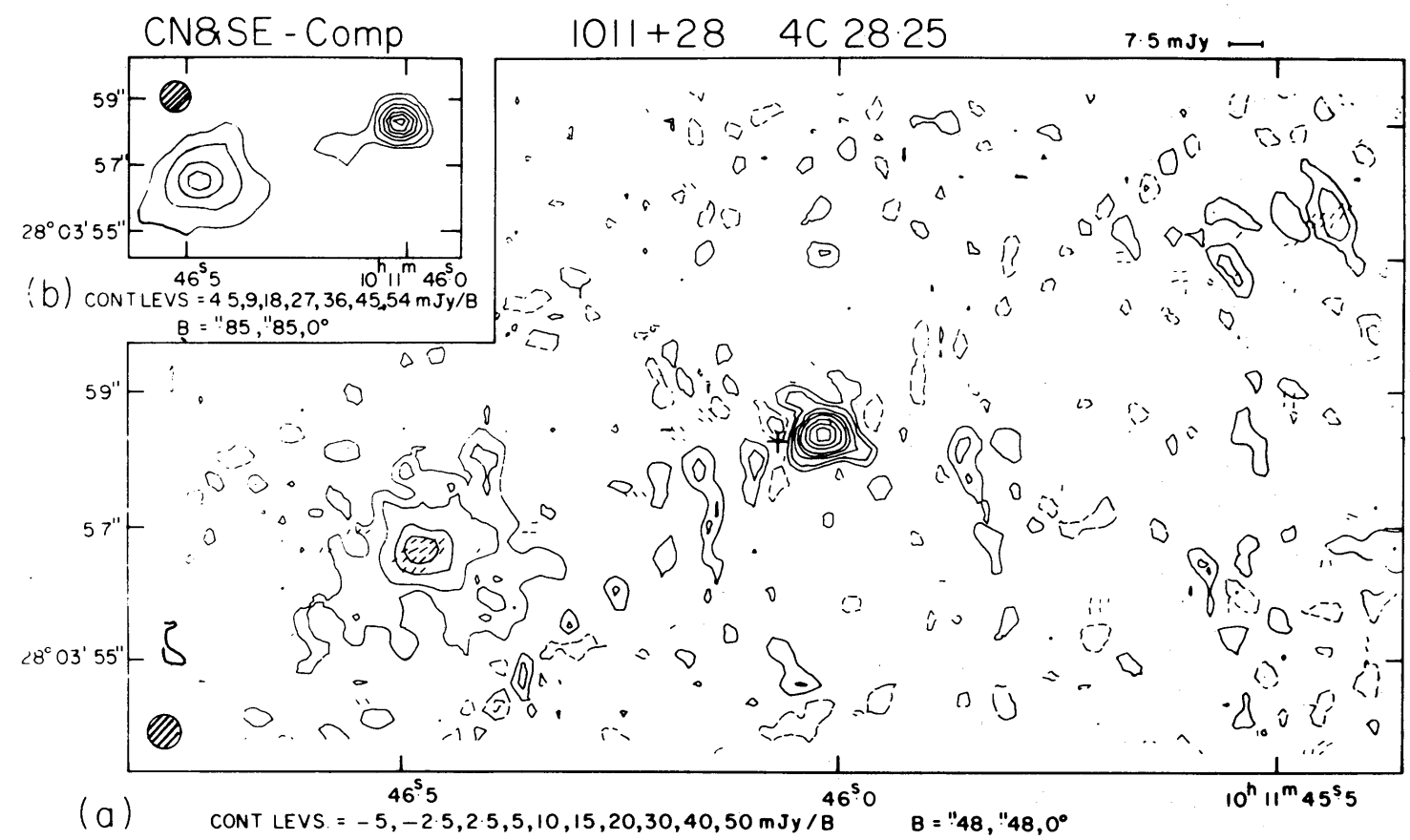

(xi)
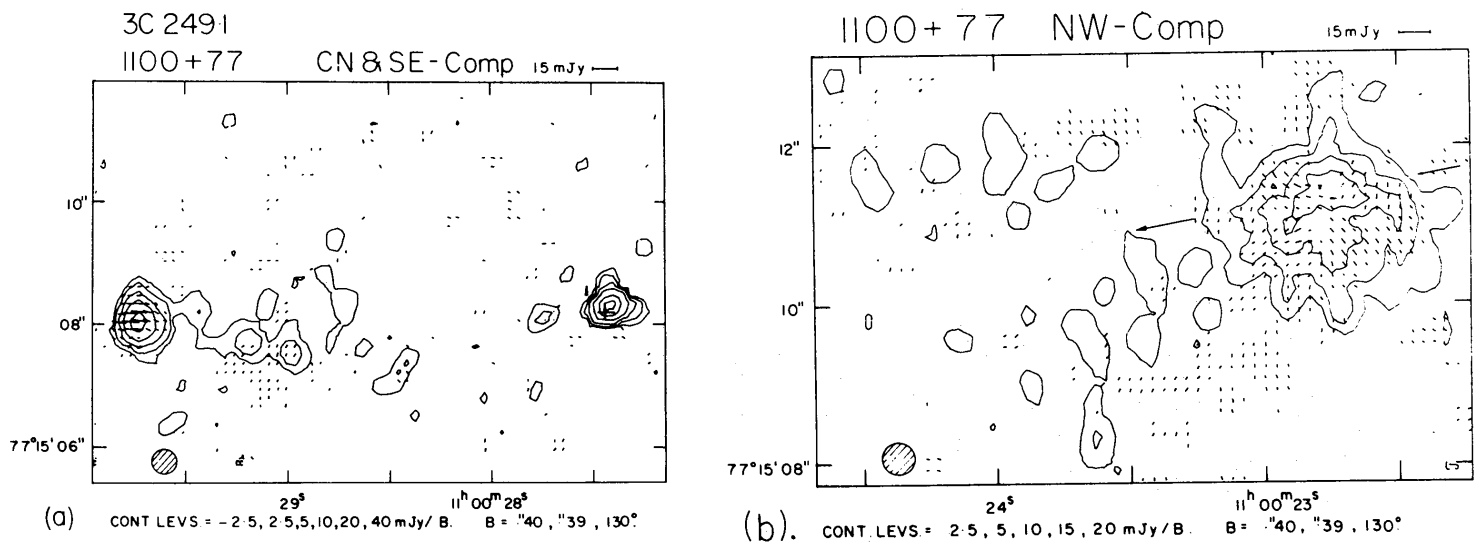

(xii)

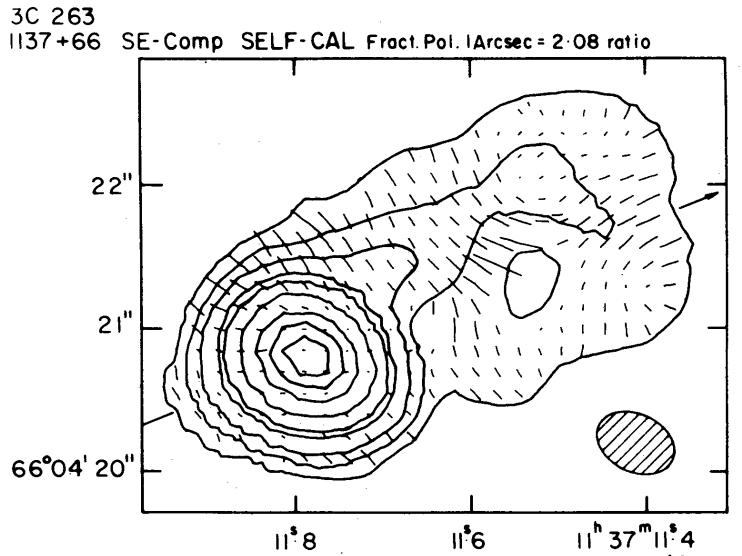

(a).CONT LEVSS. $=2 \cdot 8,6,12,28,40,80,160,240,320 \mathrm{~m} \mathrm{Jy} / \mathrm{B}$. $B=" 59, " 40,63^{\circ}$.

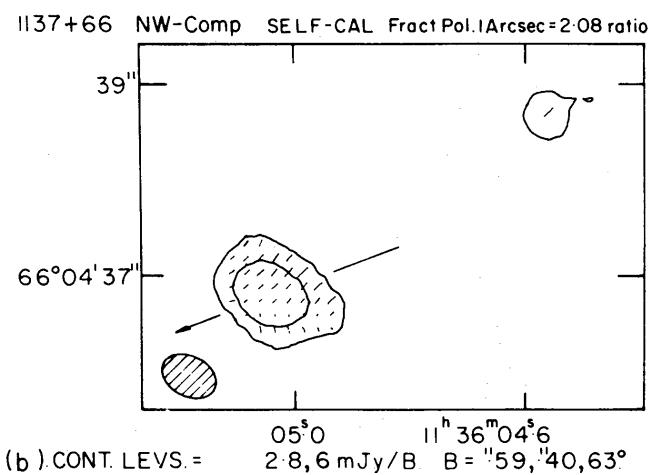

(xiii) 

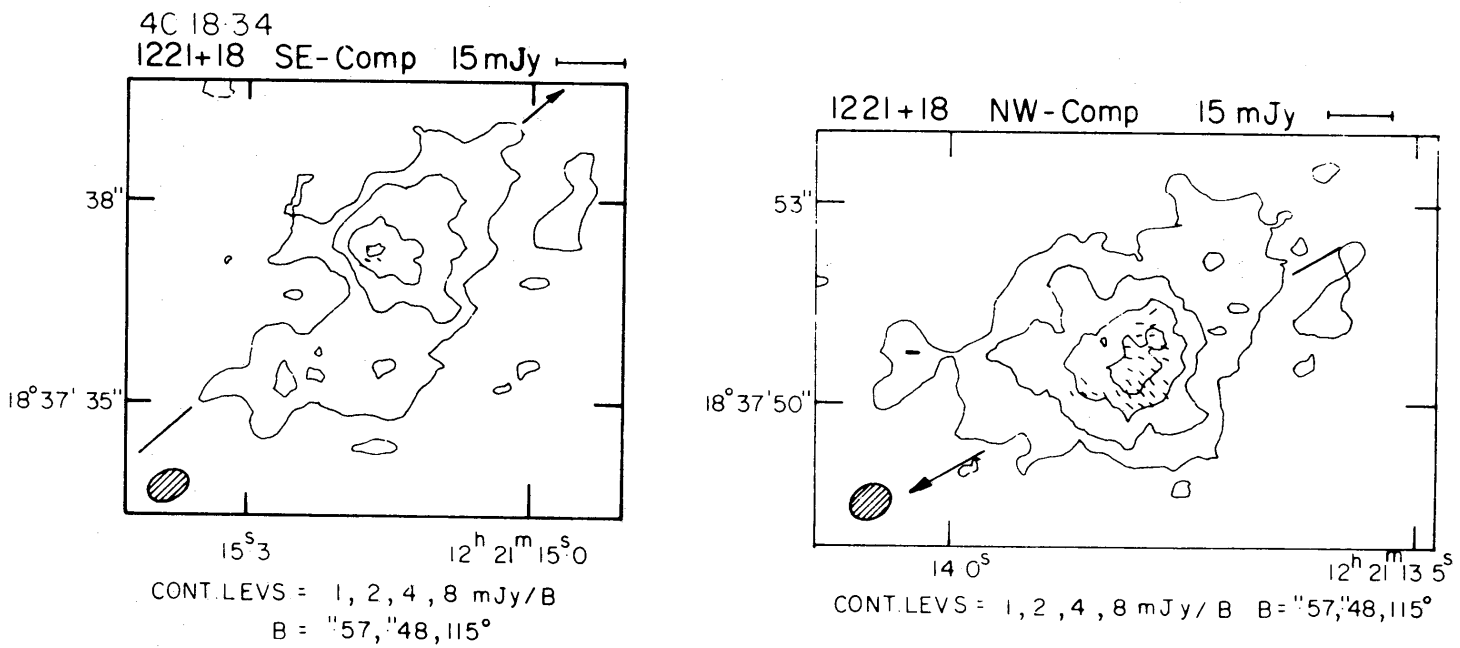

$4 C 32 \cdot 41$

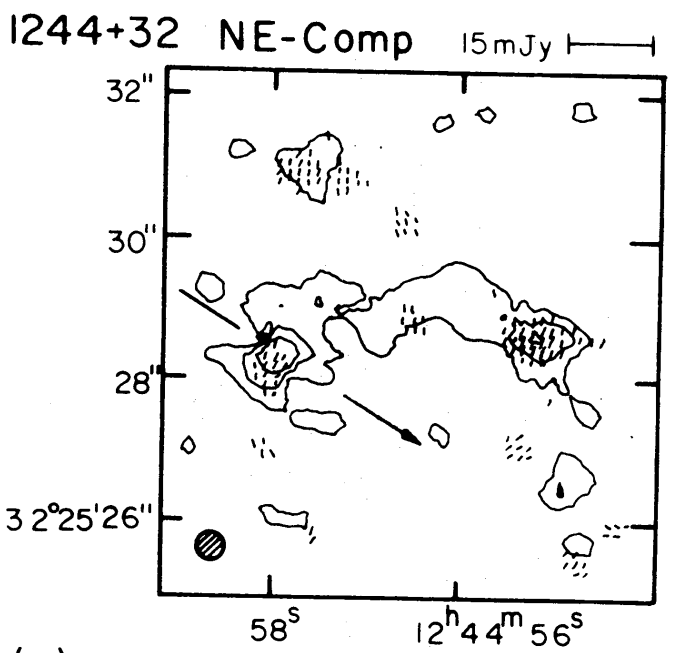

(xiv)

(a) CONT. LEVS. $=1.5,3,6 \mathrm{mJy} / \mathrm{B}$

$$
B=" 47, .45,135^{\circ}
$$

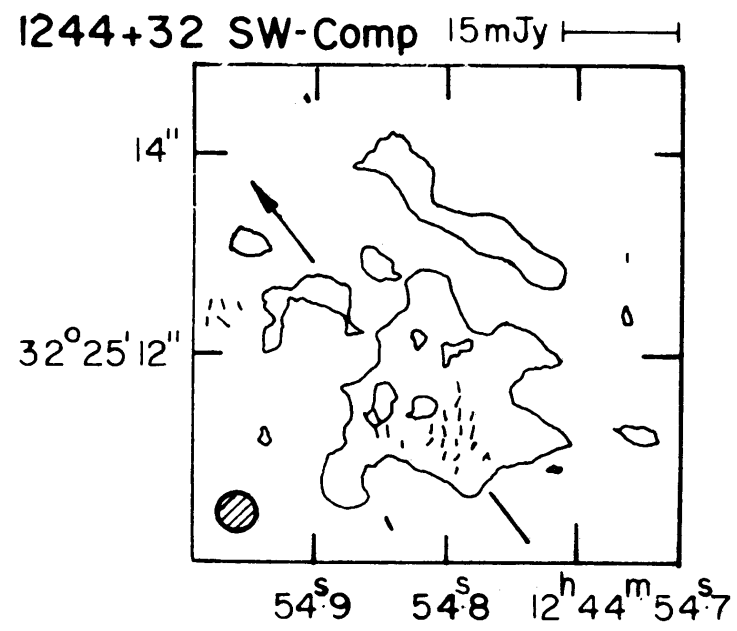

(b)

(xv)
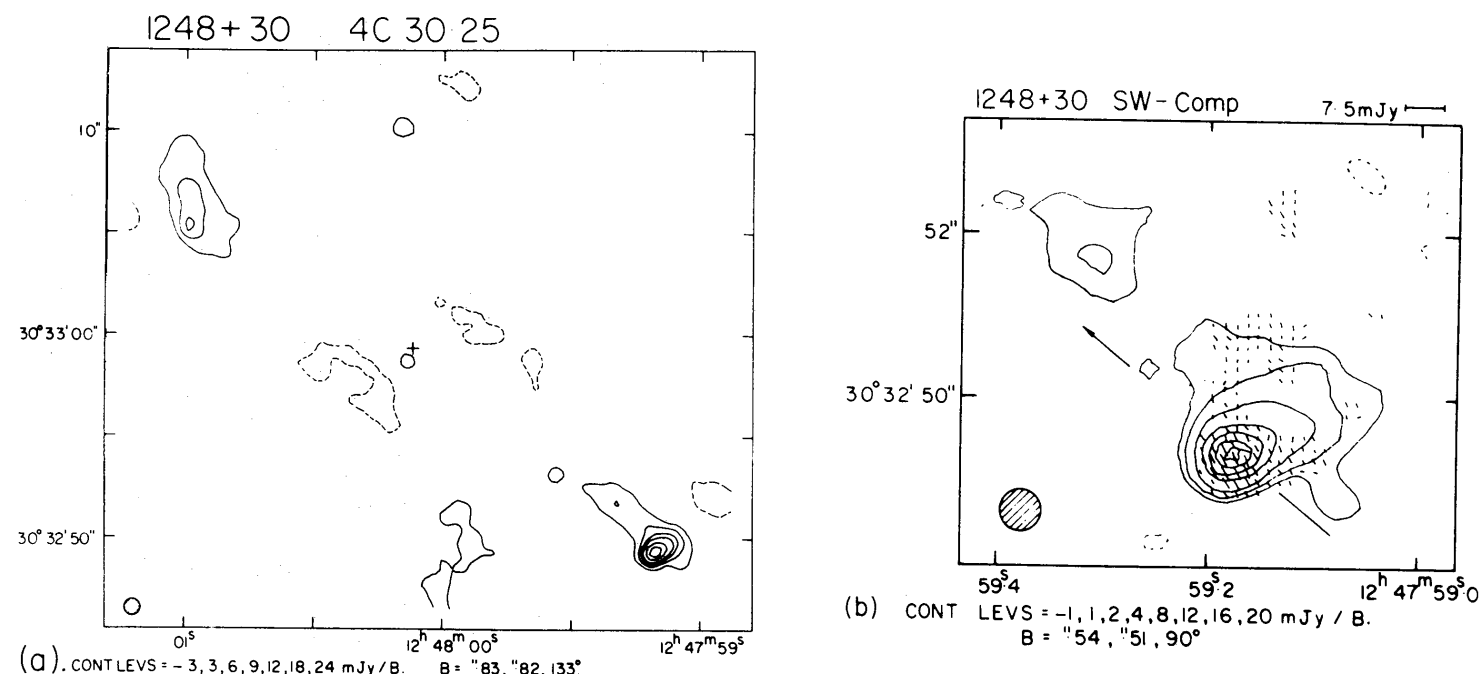

(xvi) 
Figure 1 - continued

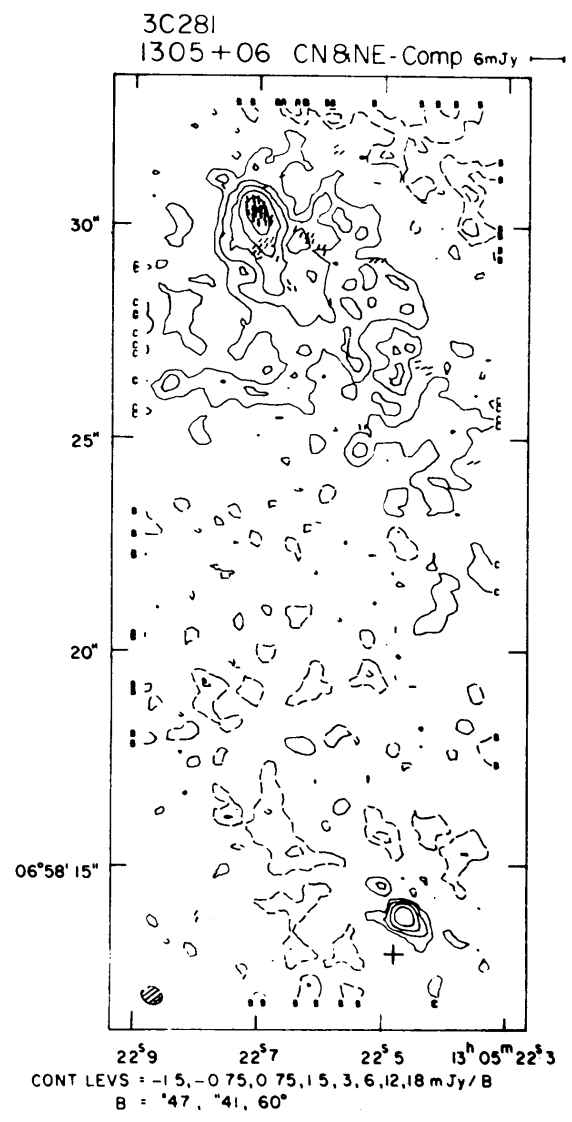

(xvii)

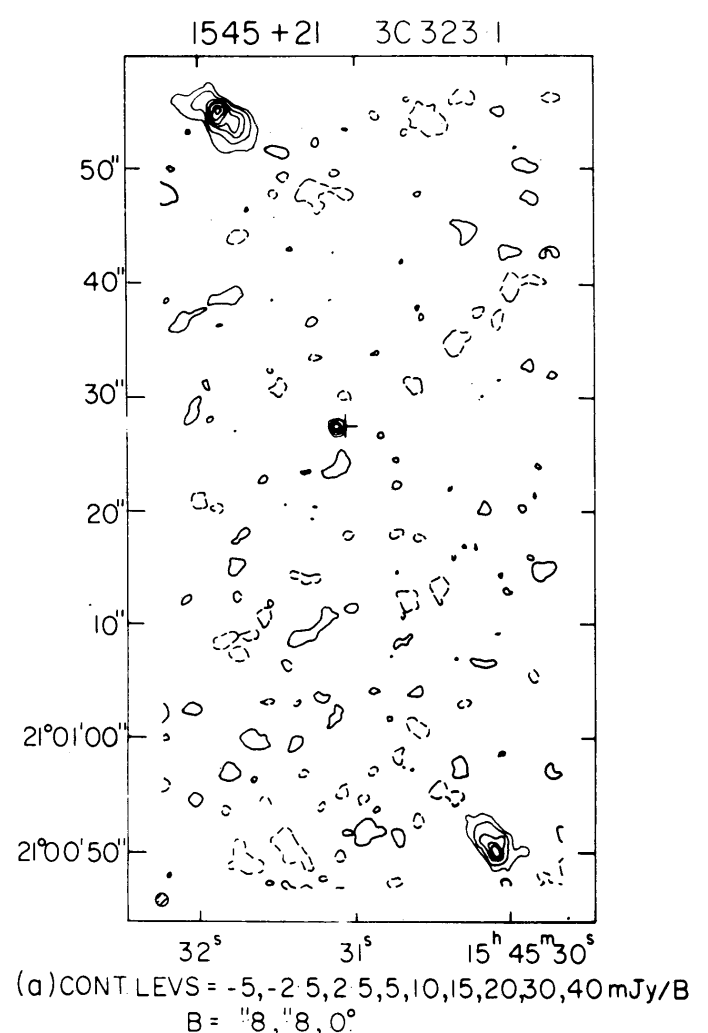

(xix)

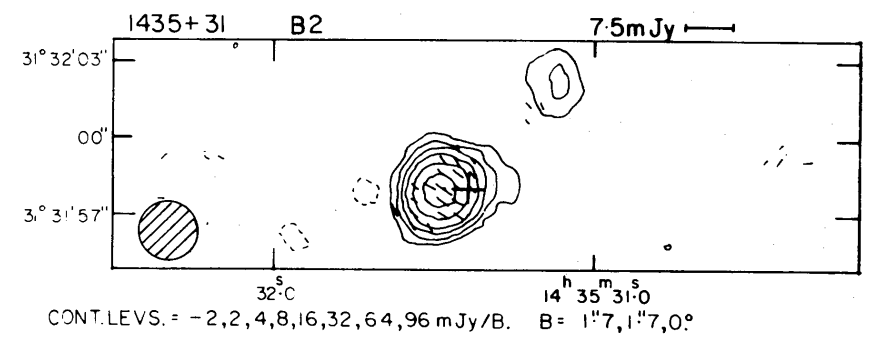

(xviii)
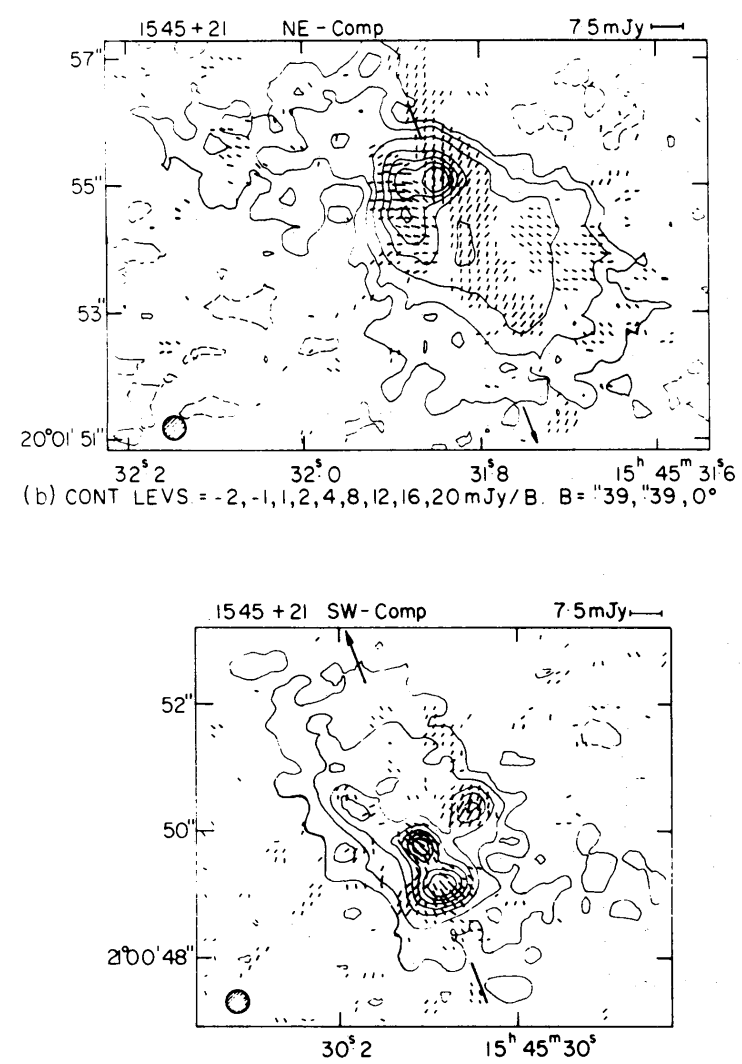

(c) CONTLEVS $=-2,-1,1,2,4,6,8,10,12 \mathrm{mJy} / \mathrm{B}$. $B=" 39, " 39,0^{\circ}$ 

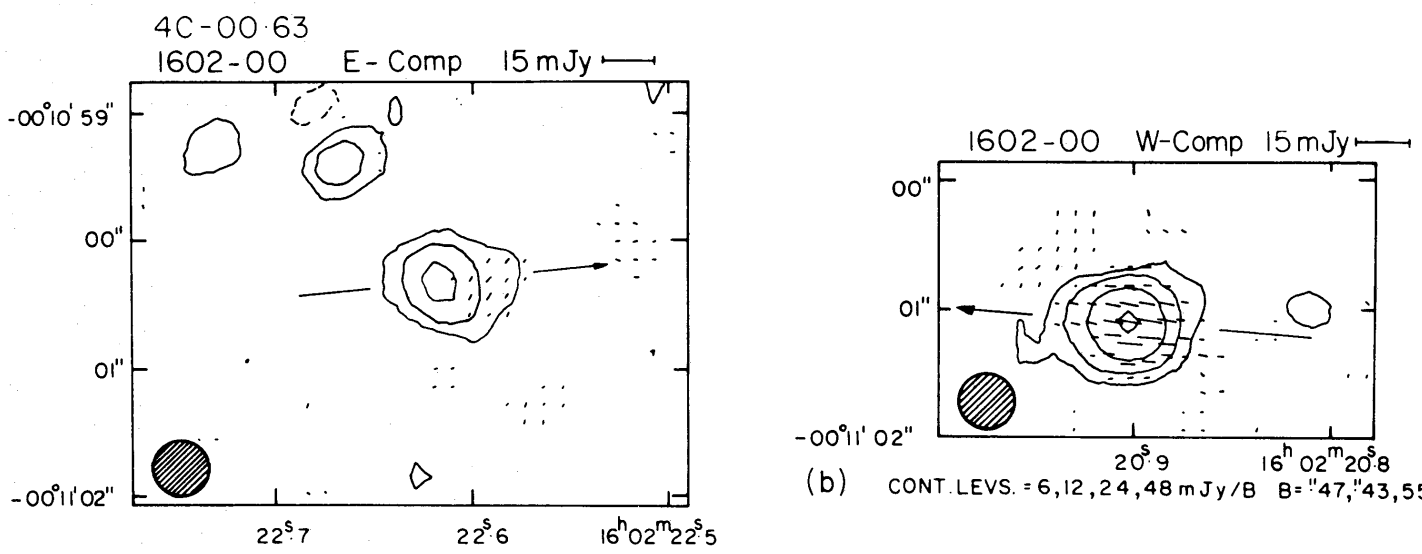

(a) CONT. LEVS $=-6,6,12,24 \mathrm{mJy} / \mathrm{B} \quad B=" 47, " 43,55^{\circ}$

(b) CONT. LEVS. $=6,12,24,48 \mathrm{~m} \mathrm{Jy} / 8 \quad B=" 47, " 43,55^{\circ}$

$(\mathrm{xx})$

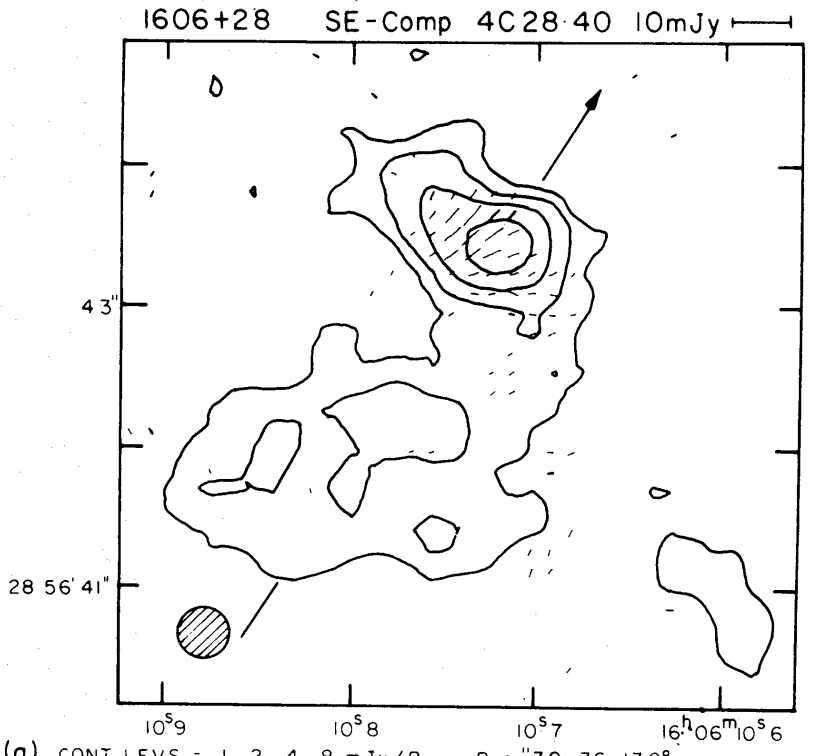

(a) CONT LeVS $=1,2,4,8 \mathrm{mJy} / 8 \quad B=" 39,36,130^{\circ}$

(xxi)

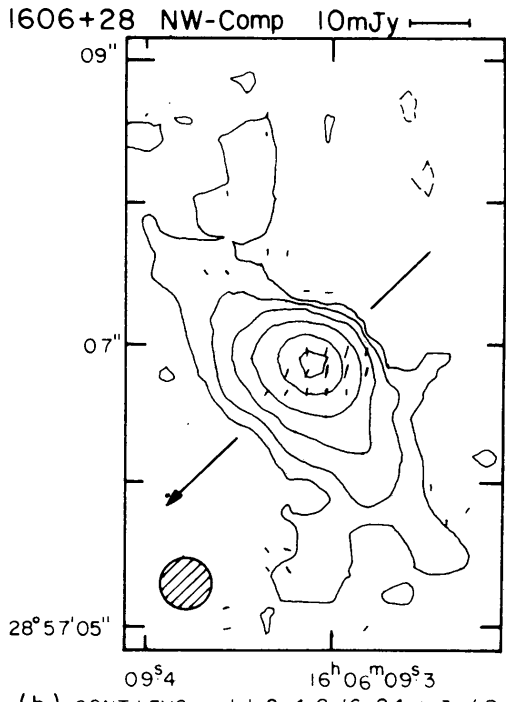

(b) CONT LEVS $=-1,1,2,4,8,16,24 \mathrm{mJy} / 8$ $B=" 39, " 36,130^{\circ}$

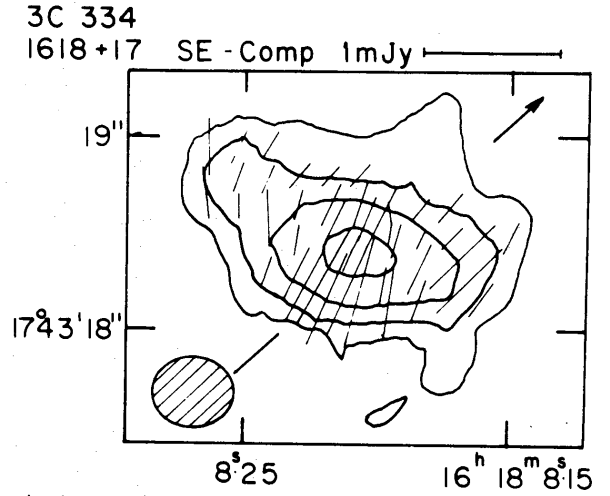

(a). CONT.LEVS. $=0 \cdot 5,1,2 \mathrm{~m} \mathrm{Jy} / \mathrm{B}$. $B=.40, " 38,96^{\circ}$.

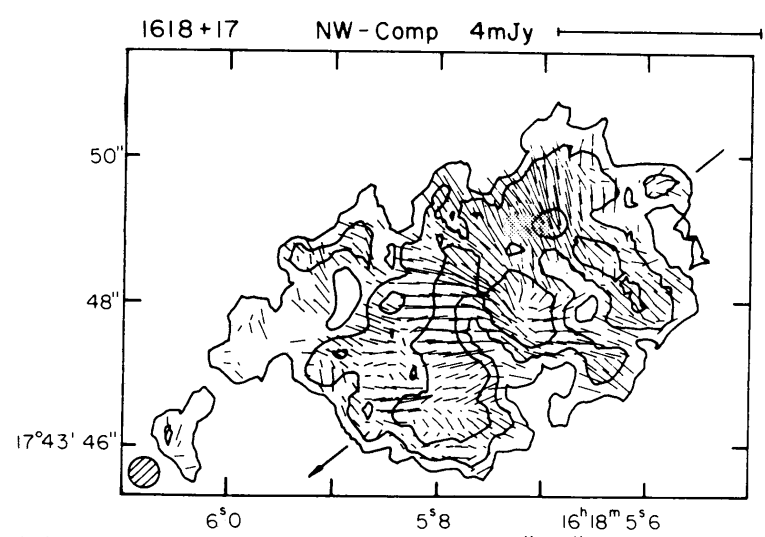

(b). CONT LEVS $=0.5,1,2 \mathrm{~m} \mathrm{Jy} / \mathrm{B} . \quad$ B $=4 " 40, " 38,96^{\circ}$ (xxii) 
Figure 1 - continued

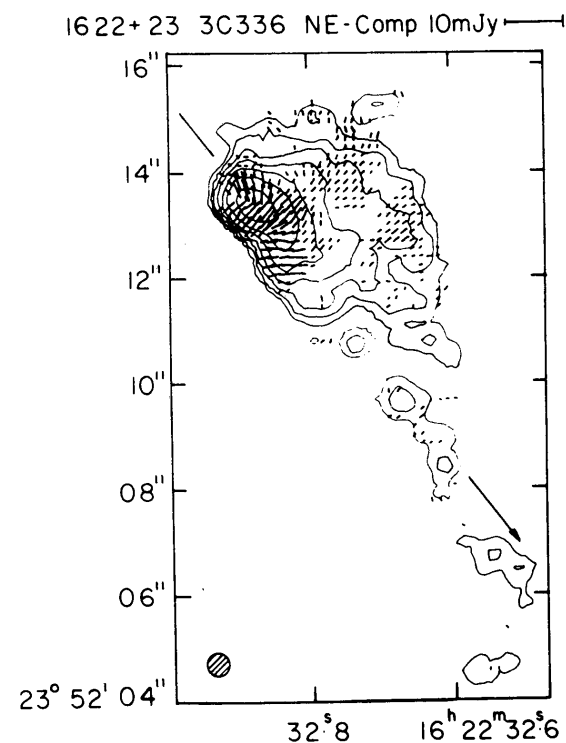

(a) CONT LEVS $=0 \cdot 5,1,2,4,8,16,32 \mathrm{mJy} / \mathrm{B}$.

$B=" 39, " 36,97^{\circ}$.

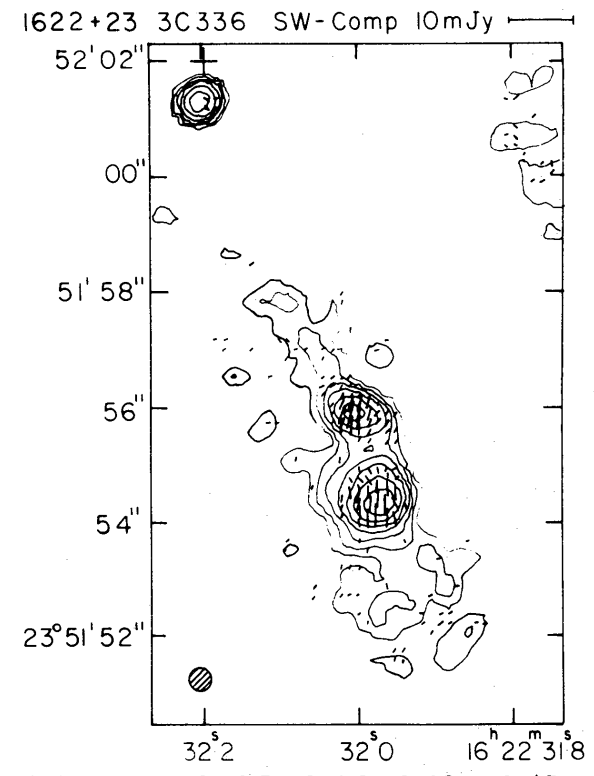

(b) CONTLEVS $=0.5,1,2,4,8,16,32 \mathrm{~m} \mathrm{Jy} / \mathrm{B}$. $B=" 39 . " 36.97^{\circ}$

(xxiii)

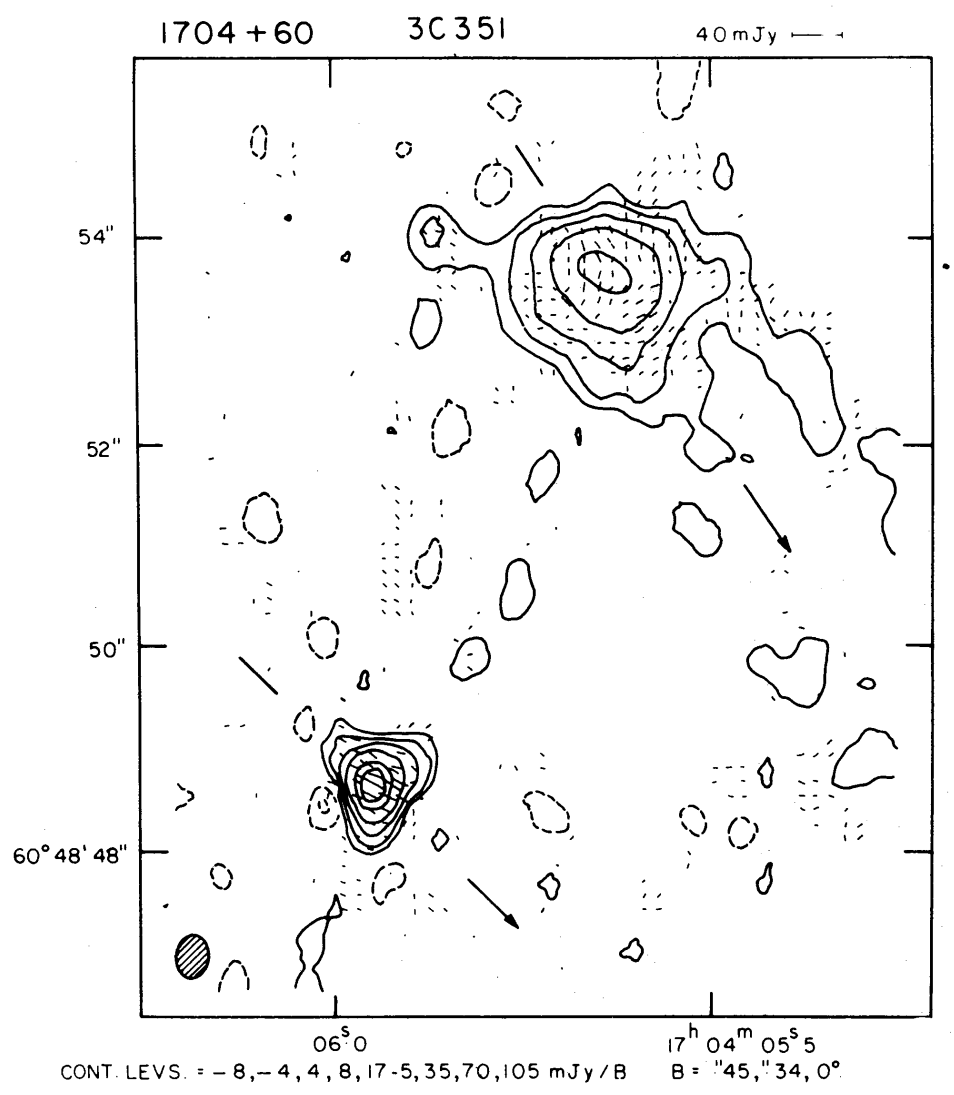

(xxiv) 

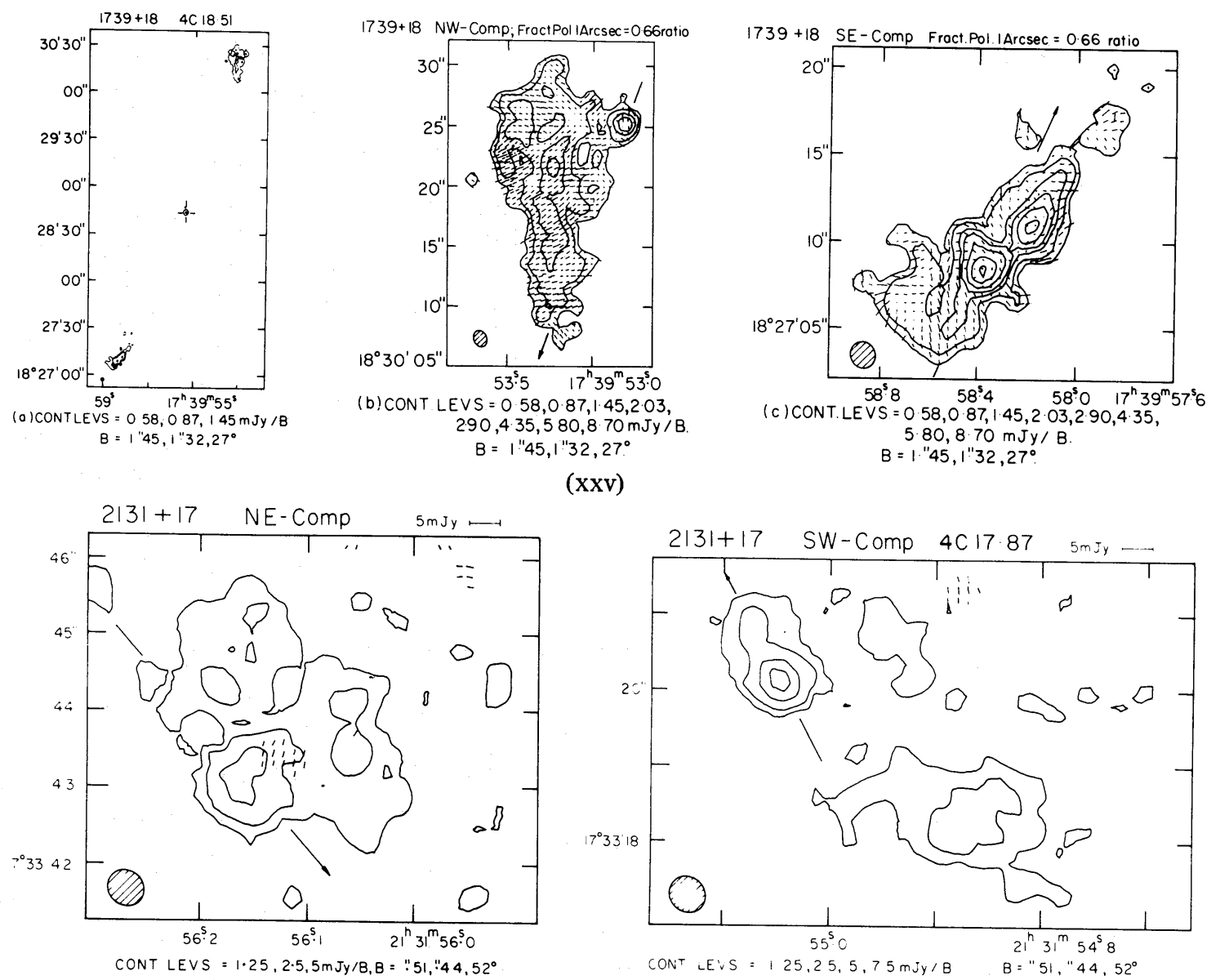

(xxv)

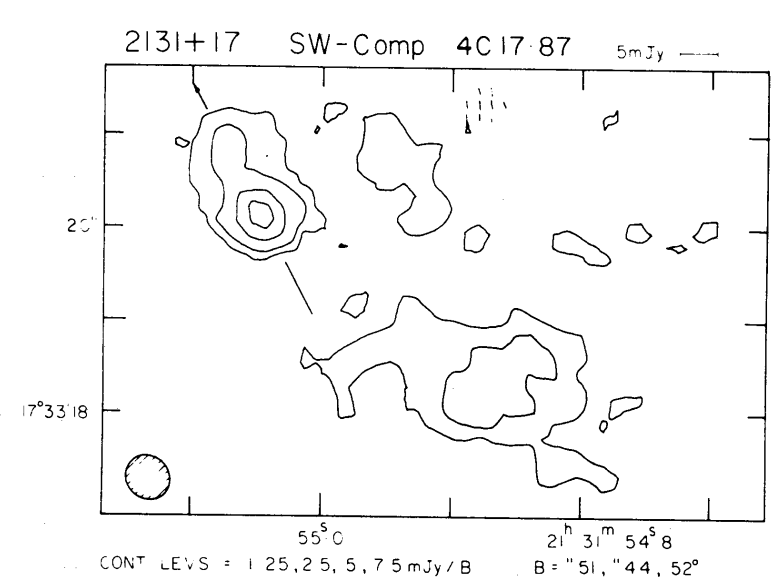

(xxvi)
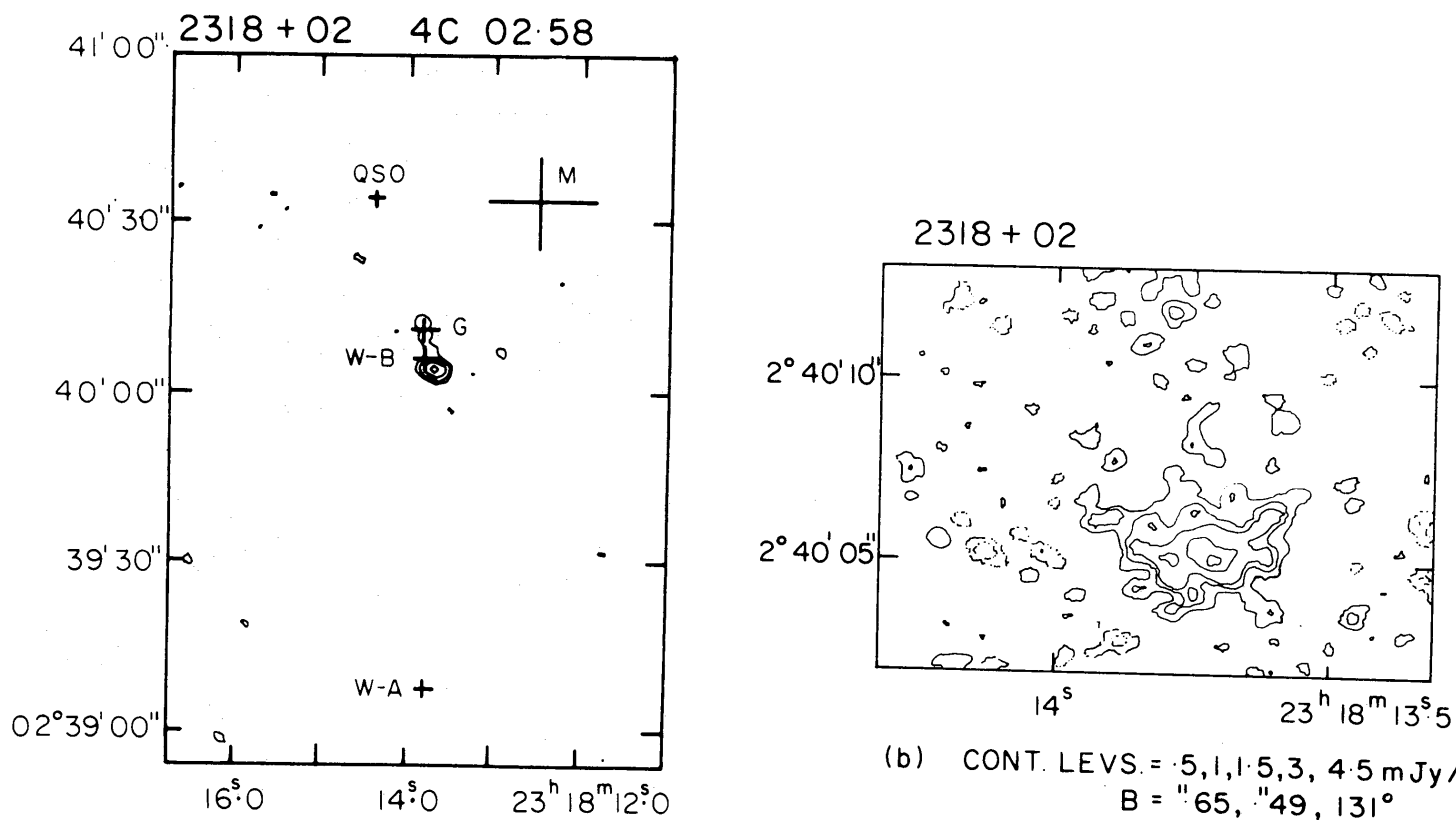

(b) CONT. LEVS $=5,1,1.5,3,4.5 \mathrm{~m} \mathrm{Jy} / \mathrm{B}$

(a). CONT. LEVS. $=2,4,8,16 \mathrm{~m} \mathrm{Jy} / \mathrm{B}$.

$$
B=" 65, .49,131^{\circ}
$$

$$
B=1 " \cdot 9,1^{\prime \prime} 32,67 \text {. }
$$

$M=$ McEwin et Cl, 1977; $G=$ Ghigo \& Owen, 1973.

$W \cdot A$ \& $W-B=$ Wills 979 , Comps $A \& B$

(xxvii) 
Figure 1 - continued
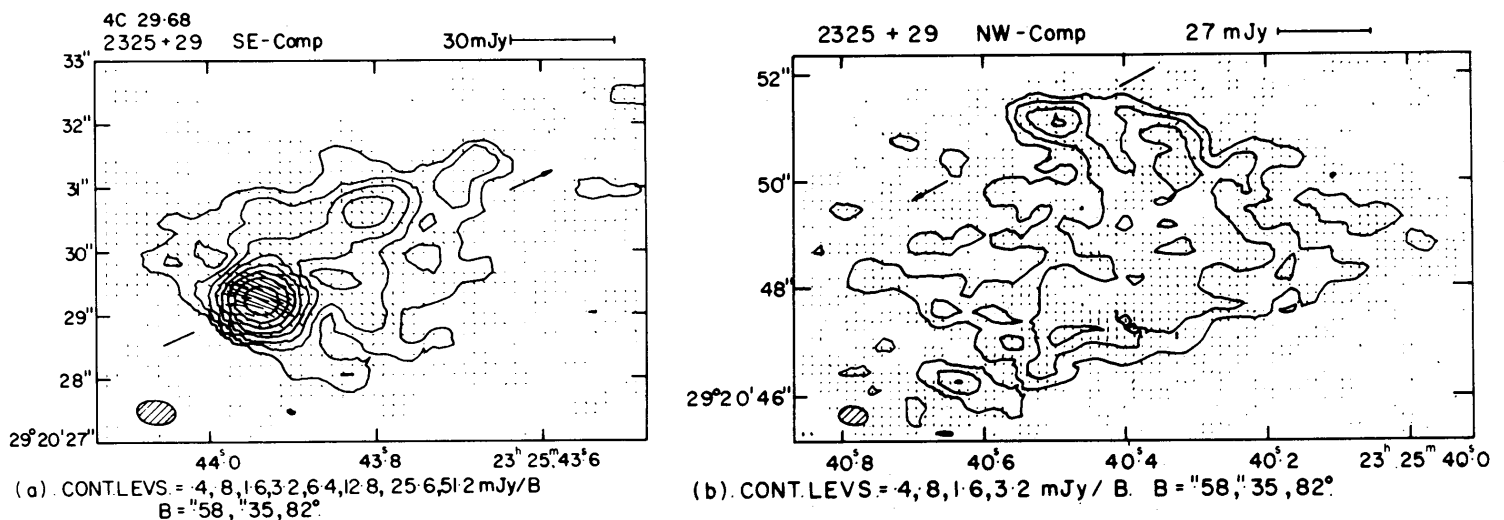

(b). CONT. LEVS $=\cdot 4 ; 8,1 \cdot 6,3 \cdot 2 \mathrm{mJy} / \mathrm{B}$. B $=" 58, " 35,82^{\circ}$.

(xxviii)

and cleaning the data with beams of $\sim 0.5,0.8$ and 2 arcsec, giving $2 \sigma$ limits of $\sim 1.5,3.5$ and $8 \mathrm{mJy}$ respectively.

$0835+58$. The SW2 component is more diffuse than the SW1 component. Using an interferometer with a base line of $1.26 \times 10^{6}$ wavelengths, Kapahi \& Schilizzi (1979) detected a compact hotspot in the SW component which has a maximum correlated flux density of $317 \mathrm{mJy}$ at $1.4 \mathrm{GHz}$; assuming a spectral index of about -1 , we estimate its peak flux density to be about $100 \mathrm{mJy}$ at $4.87 \mathrm{GHz}$ and its size to be $<0.1$ arcsec. This implies that the minimum energy density of the SW1 hotspot has a value appreciably higher than the $3 \times 10^{-8} \mathrm{erg} \mathrm{cm}^{-3}$ given in Table 2 .

$0903+16$. The radio structure of this quasar is considerably distorted, similar to that of low-radio luminosity sources located in clusters (Miley 1980). Gopal-Krishna \& Swarup (1977) have compared occultation observations at $327 \mathrm{MHz}$ made by Kapahi, Gopal-Krishna \& Joshi (1974) with the $5 \mathrm{GHz}$ map by Pooley \& Henbest (1974). This comparison indicates that there is diffuse structure with a rather steep spectrum between the two outer lobes.

$0911+05$. Our cleaned VLA map shows only a central component of peak flux density $\sim 48 \mathrm{mJy}$ located near the quasar; the maximum visibility at antenna spacings of about 6000 wavelengths is found to be about $90 \mathrm{mJy}$. Miley \& Hartsuiker (1978) report a double structure at $6 \mathrm{~cm}$ with a flux density of $\sim 100 \mathrm{mJy}$ in each of the outer components and an overall size of $95 \pm 20$ arcsec. Our observations indicate that their result could be in error, perhaps due to the low declination. Wardle \& Miley (1974) report $0911+05$ to be a double source with LAS of 55 arcsec and component sizes of $\sim 3 \operatorname{arcsec}$ for the stronger and $\sim 7$ arcsec for the weaker. Thus there is uncertainty about the angular structure of this low-redshift quasar and it is not considered further in this paper.

$0932+02$. The source has a highly asymmetric structure, with the NE lobe located relatively close to the QSO. The resolution of earlier observations was insufficient and it was wrongly classified as a D2 source by Kapahi (1981).

$0957+00$. A smoothed and cleaned map with $1.7 \times 1.7 \operatorname{arcsec}^{2}$ resolution shows an additional component $\sim 5$ arcsec east and 2 arcsec south of the SE component producing $\sim 30$ per cent of the flux of the SE component given in Table 1.

$1004+13$. The outer components of this source are fully resolved out at $5 \mathrm{GHz}$ to $<2 \mathrm{mJy} /$ beam with a resolution of about $0.8 \times 0.7 \mathrm{arcsec}^{2}$. A weak central component is present with a peak flux density of $\sim 10 \mathrm{mJy}$. VLA observations by Fomalont (1981) at $5 \mathrm{GHz}$ and Westerbork observations by Miley \& Hartsuiker (1978) at $5 \mathrm{GHz}$ with resolutions of about $4.3 \times 3.3$ and $6 \times 26 \operatorname{arcsec}^{2}$ respectively show that this source has a prominent 
radio lobe located about 70 arcsec to the west of the central component and a radio jet flaring up to a diffuse lobe about 45 arcsec to the east.

$1011+28$. By combining VLA data at $5 \mathrm{GHz}$ with the interferometric measurements made at 2.7 and $8 \mathrm{GHz}$ by Potash $\&$ Wardle (1979), it is seen that the central component has an extended feature of steep spectrum, superimposed on a compact component whose flux density increases towards higher frequency. The full source has a spectral index of -0.96 between 0.4 and $1.4 \mathrm{GHz}$ and -0.27 between 1.4 and $5 \mathrm{GHz}$.

$1100+77$. The VLA map indicates a possible radio jet between the quasar and the eastern hotspot. The NW component seems to have a circumferential magnetic field. A recent MERLIN map at $408 \mathrm{MHz}$ made at Jodrell Bank shows a weak diffuse component farther out from the SE component at a distance similar to that of the NW component (Lonsdale \& Morison 1983; Miley \& Hartsuiker 1978).

$1221+18$. The radio lobes of this source are relatively relaxed and there are no prominent hotspots at their extremities. The degree of polarization is small.

$1244+32$. A curved elongated structure is present in the NE lobe, lying between the outermost hotspot and the strong central component of the source. Since the cleaning of the source was poor, our values of the flux densities of the components are not included in Table 1.

$1248+30$. In a manner similar to the SW component, the NE lobe is also bent towards $\mathrm{NW}$ as seen in a map obtained with a resolution of $1.5 \times 1.4 \mathrm{arcsec}^{2}$.

$1305+06$. The SE component reported by Miley \& Hartsuiker (1978) seems to be diffuse, because it is resolved out in the VLA map.

$1435+31$. The source seems to have a D2 structure, as is also seen by Fanti et al. (1977) who report its LAS as $12 \mathrm{arcsec}$. From maps made with resolutions of $0.4 \times 0.4 \mathrm{arcsec}^{2}$ (see Table 1) and $1.7 \times 1.7 \operatorname{arcsec}^{2}$ (Fig. 1 xviii), it is seen that the central component has an unresolved compact core of about $94 \mathrm{mJy}$ with diffuse emission of about $13 \mathrm{mJy}$. In addition, there exists a NW component whose flux density is greater than $11 \mathrm{mJy}$. Using a $1.7 \times 1.7$ $\operatorname{arcsec}^{2}$ resolution, it is found that the central component has a polarized flux density of $1.8 \mathrm{mJy}$ at $54^{\circ} \mathrm{PA}$. The spectral index of the source between $408 \mathrm{MHz}$ and $5 \mathrm{GHz}$ is -0.27 .

$1545+21$. In the map by Pooley \& Henbest (1974) made with the Cambridge $5-\mathrm{km}$ radio telescope at $5 \mathrm{GHz}$, the $\mathrm{NE}$ radio lobe is appreciably extended towards the west. The redshift of the quasar is 0.264 which is close to that of a nearby cluster of galaxies (Oemler, Gunn \& Oke 1972; Baldwin 1975).

$1602+00$. This QSO is clearly a triple source, although Wills (1979) reported it to be only a double. The flux density of the central component given by him is likely to be the sum of the values for our central and eastern components. The quasar has a strong central component and is known to be a variable source at $\mathrm{cm}$ wavelengths (Wall 1972; McEwan, Browne \& Crowther 1975).

$1606+28$. A map with a $2 \times 2 \operatorname{arcsec}^{2}$ beam shows a central component with $6 \mathrm{mJy}$ peak value at about 3 or $4 \mathrm{rms}$ level, but a map with a $0.4 \times 0.4 \operatorname{arcsec}^{2}$ beam shows only a peak value of $<2 \mathrm{mJy}$. This indicates that the central component may be extended without a compact region, which is unusual.

$1618+17$. A radio jet is seen between the SE component and the QSO by Wardle \& Potash (1982), and their map also reveals that the direction of energy-flow bends by about $90^{\circ}$ beyond the SW hotspot to form a diffuse lobe. This is not seen in our map because of the limited dynamic range.

$1704+608$. A low-resolution map made with a $20 \times 23 \operatorname{arcsec}^{2}$ beam at $1.4 \mathrm{GHz}$ shows a weak south-western component (Mackay 1969; Kronberg, Clarke \& van den Bergh 1980), and a weak central component is seen by Riley \& Pooley (1975), Laing (1981) and Kronberg et al. (1980). 
$1739+18$. The VLA 'A' configuration with a smoothed beam of $0.74 \times 0.65 \operatorname{arcsec}^{2}$ shows only a compact component of size $<0.3 \mathrm{arcsec}$ with a peak of $21 \mathrm{mJy}$ and a total flux density about $31 \mathrm{mJy}$. The map given in Fig. 1(xxv) was observed with the 'B' configuration of the VLA in 1982 August by D. J. Saikia. It has two extended lobes with weak hotspots and has a ' $\mathrm{C}$ '-shaped geometry.

$2318+02$. Fig. 1(xxviia) shows a map made with a beam of $1.9 \times 1.32 \operatorname{arcsec}^{2}$. The feature seen in the map has a compact component with an extended tail, and corresponds to the component B of Wills (1979). No other radio component with a brightness above $2 \mathrm{mJy} /$ beam area is seen within $\pm 60 \mathrm{arcsec}$ of this feature. The QSO lies about 34 arcsec to the north-east of this feature. The centroid of the radio source at $365 \mathrm{MHz}$ (Ghigo \& Owen 1973 ) is only $\sim 7 \mathrm{arcsec}$ to the north of the feature seen by us. At $365 \mathrm{MHz}$, Cotton, Owen \& Ghigo (1975) report it to be either a double radio source of separation $40 \pm 15 \operatorname{arcsec}$ along PA $170^{\circ} \pm 10^{\circ}$, or an elliptical source with a size $51 \pm 17 \operatorname{arcsec}$ in PA $=106^{\circ} \pm 15^{\circ}$. The size of the double radio source given by Wills (1970) is 68 arcsec. These data might indicate that the radio source is a D2 quasar. In that case the linear size of the radio source would be unusually large for a quasar at $z=1.968$. Also D2s tend to have prominent central components associated with the quasar (Kapahi 1981) while here a central component is not detected. However, it is possible (Wills 1979) that the QSO may not be associated with this radio source. To determine the overall structure and identification of this radio source, it should be mapped with a lower resolution than used here. For the present, we do not consider it as a confirmed quasar.

\subsection{DERIVED PARAMETERS OF QUASARS}

Table 2 gives derived physical parameters for 31 quasars. Data for quasars $0017+15$ (3C9) and $1258+40$ (3C 280.1) given by Swarup et al. (1982a) are also included, but not the sources $0911+05$ and $2318+02$ described in Section 3.3, for the reasons given there. Values of $q_{0}=0$ and $H_{0}=50 \mathrm{~km} \mathrm{~s}^{-1} \mathrm{Mpc}^{-1}$ have been assumed. Table 2 is arranged as follows:

(1) The source name.

(2) $3 \mathrm{C}, 4 \mathrm{C}$ or $\mathrm{B} 2$ name.

(3) Redshift.

(4) The flux density at $178 \mathrm{MHz}, S_{178}$. The values have been taken from Jenkins, Pooley \& Riley (1977) and Gower, Scott \& Wills (1967), and have been multiplied by correction factors of 1.05 and 1.11 respectively as determined by Kühr et al. (1979). For B2 sources, the flux densities were extrapolated from 408 to $178 \mathrm{MHz}$ using the known values for the spectral indices.

(5) The radio luminosity, $P_{178}$, at the emitted frequency of $178 \mathrm{MHz}$. This is derived from $S_{178}$ and the value of the low-frequency spectral index, the latter taken mostly from Hooley et al. (1978) and Wills \& Lynds (1978). For some cases the spectral index was found by plotting the known flux densities at different frequencies.

(6) Component designation.

(7) Estimated flux density, $S_{\mathrm{h}}$, of the hotspot derived by multiplying its observed peak flux density (Table 1, column 8) by the ratio of the observed area of the hotspot (based on the measured FWHP before deconvolution) to that of the VLA beam used for mapping the source. An asterisk (*) indicates that only the peak value is given and is the same as in column 8 of Table 1 ; this is the case for central components and very weak hotspots.

(8) The first row against each source gives the total flux density at $5 \mathrm{GHz}, S_{\mathrm{t}}$. The value given against each component is its estimated flux density, $S_{1}$ (Table 1, columns 9 or 10). 


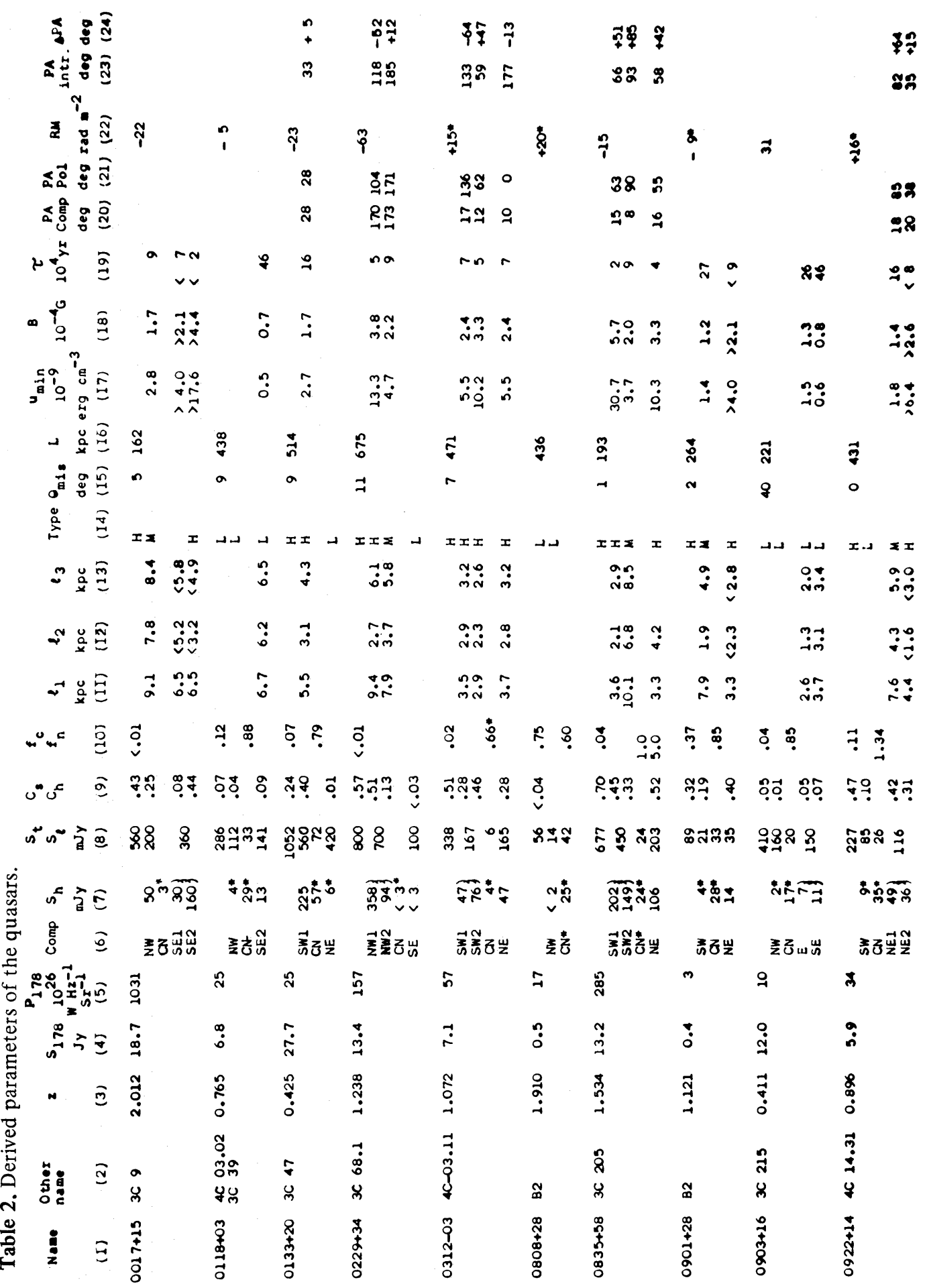




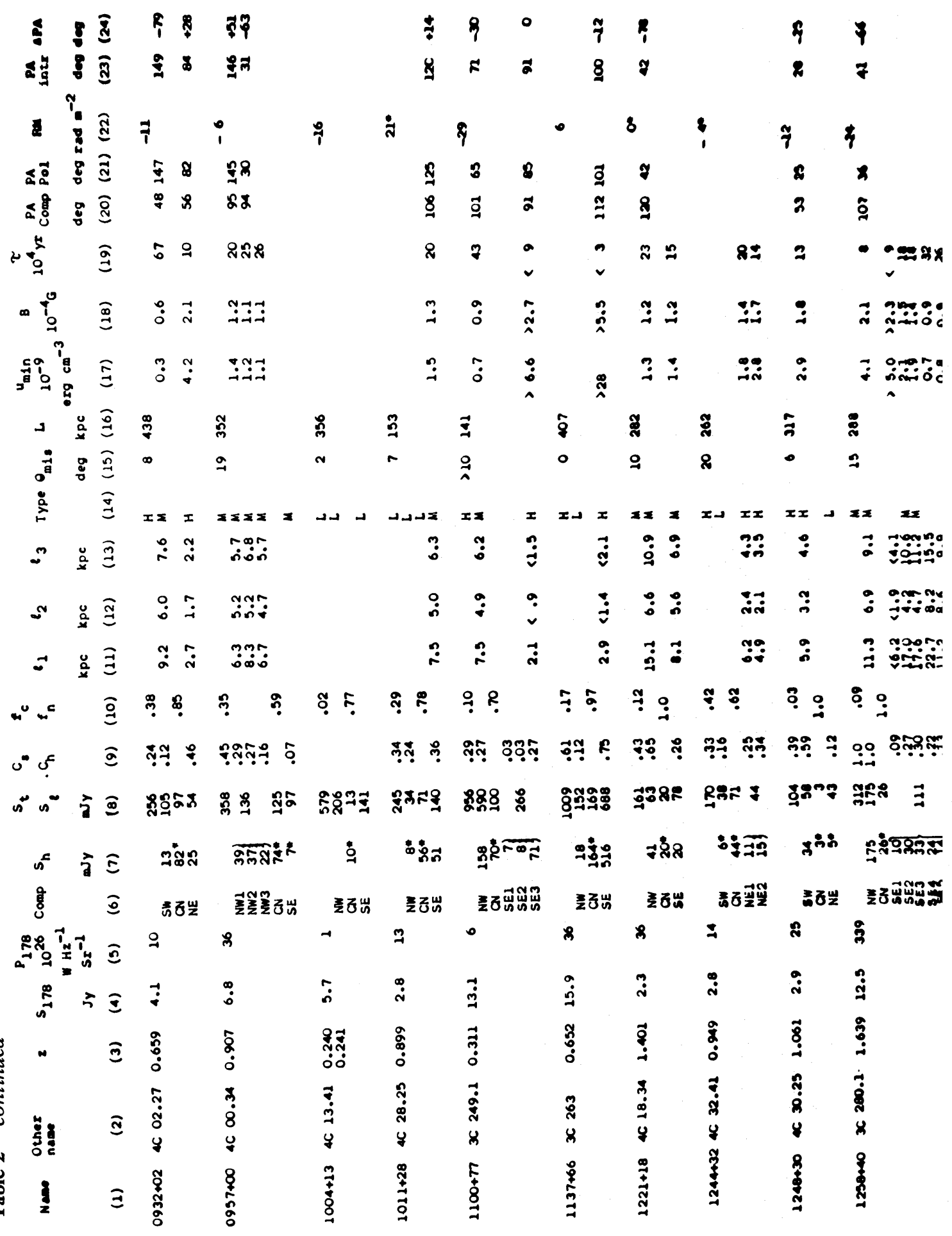




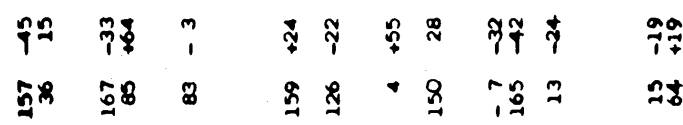

$\stackrel{\vdots}{\vdots}$

$$
i
$$

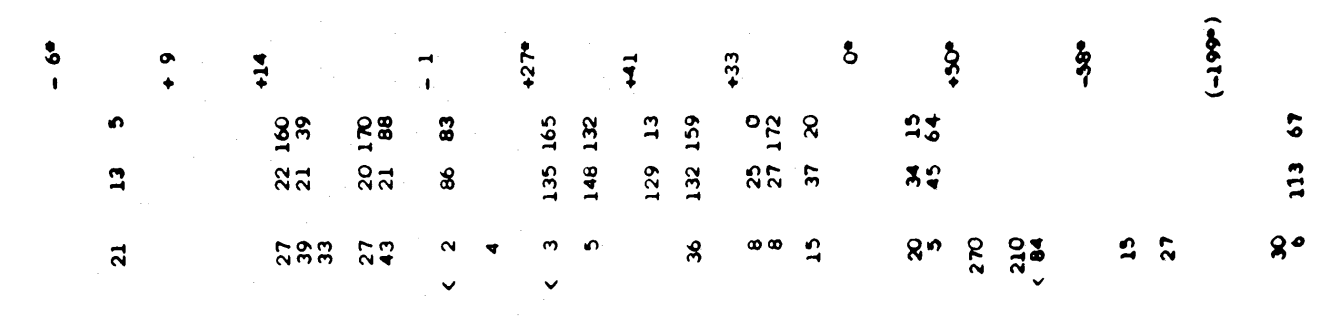

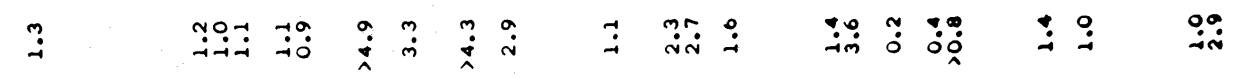

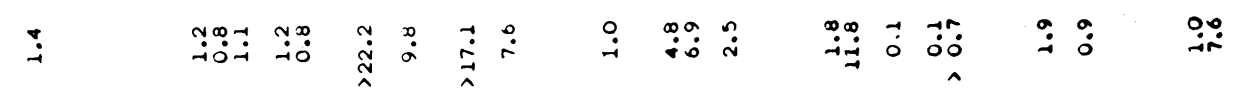

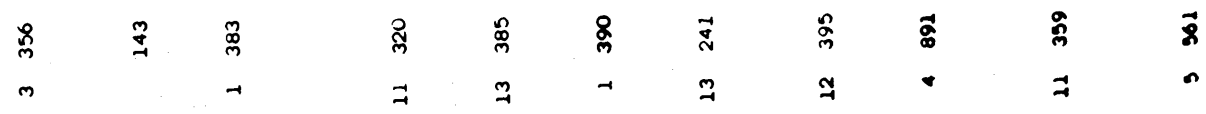

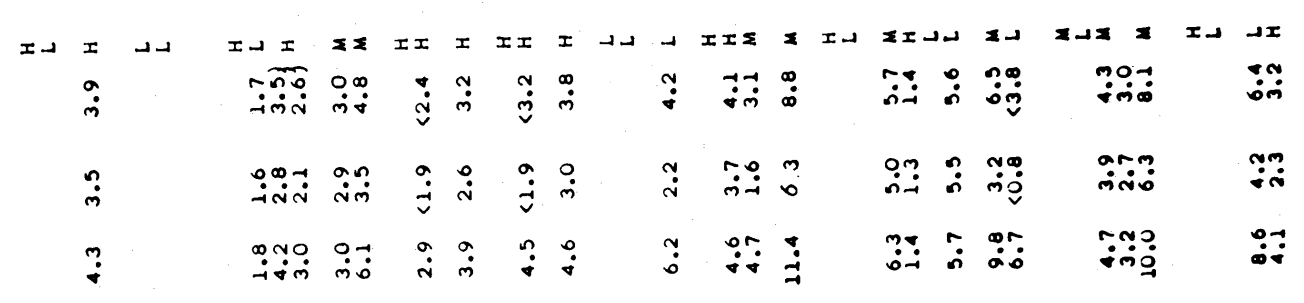

○.

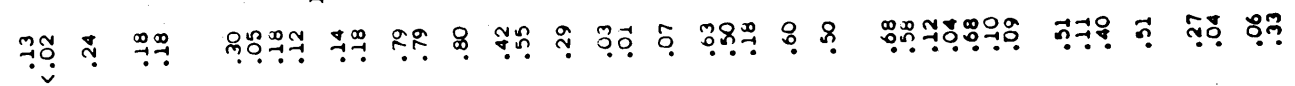

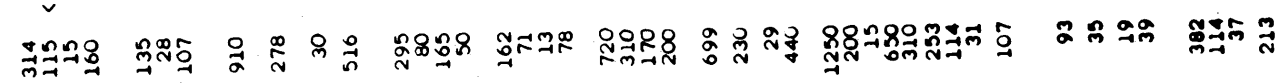

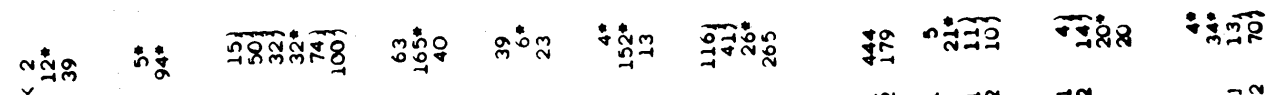

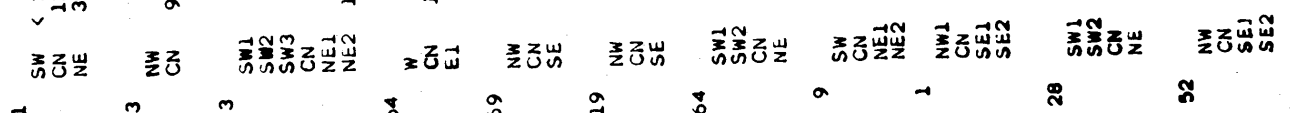

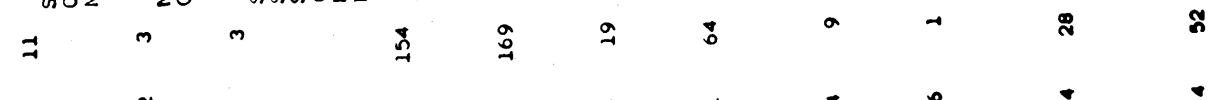

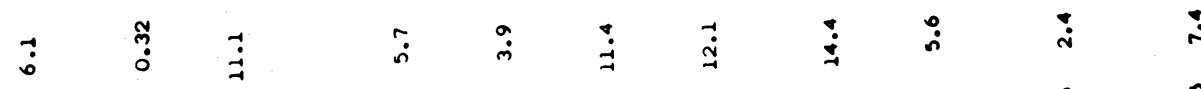

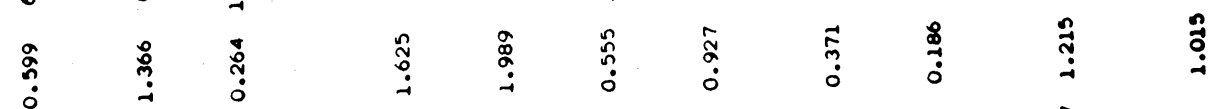

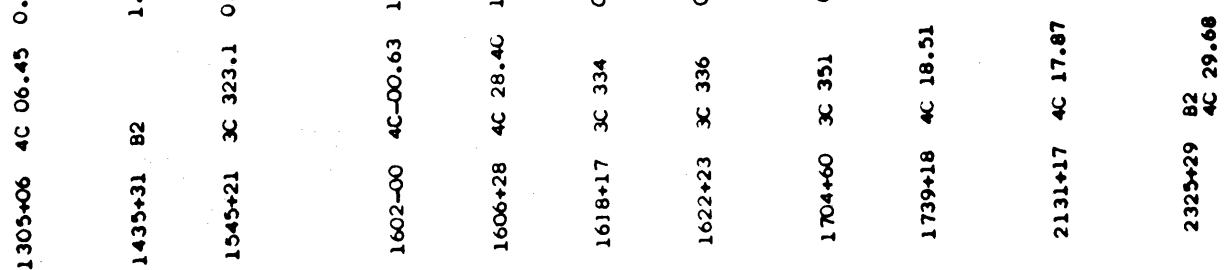


(9) The first row against each source gives a 'compactness parameter', $C_{\mathrm{s}}$, equal to the fraction of the total flux density in various hotspots to the integrated flux density of the source minus the value of the central component. In the subsequent rows for each source, the fraction, $C_{\mathrm{h}}$, of the flux density of the hotspot to that of the corresponding radio lobe is given.

(10) The first row gives the fraction, $f_{\mathrm{c}}$, which is the ratio of the flux density of the central component to that of the entire source as measured at $4.87 \mathrm{GHz}$. The value, $f_{\mathrm{n}}$, given in the row marked $\mathrm{CN}$ (central component), is the ratio of the peak value to the total flux density of the central component. It is generally $<1$ as expected, but is $>1$ for three sources $(0922+14 ; 1545+21 ; 2131+17)$ perhaps arising from errors of measurement.

(11), (12) and (13) Linear sizes $l_{1}$ and $l_{2}$ of hotspots derived from the observed FWHP values $\psi_{1}$ and $\psi_{2}$ (Table 1) along their major and minor axes respectively. Column 13 gives the mean sizes $l_{3}$.

(14) Classification of the quasars and the type of each hotspot, according to the scheme described in Section 4.1

(15) The 'mis-alignment' angle between the lines joining the central component to the brightest hotspots on opposite sides of the quasars or to the centre of the extended lobe if no prominent hotspot is seen.

(16) The physical projected size of the quasar derived from its LAS.

(17) and (18) The minimum energy density, $u_{\min }$, and the equipartition value of the magnetic field, $B$, using the equations given by Pacholczyk (1970). The volume of the hotspot has been taken as $\pi l_{1} l_{2}^{2} / 4$, assuming a cylindrical geometry. A filling-factor of unity is assumed for the volume occupied by the relativistic particles and magnetic fields. The ratio of the energy of protons to that of electrons is taken as unity. A spectral index of -0.65 has been assumed for all the hotspots, as the spectral index of a hotspot is generally flatter than that of the entire radio source (Laing 1981).

(19) The lifetime, $\tau_{\mathrm{s}}$, considering both the synchrotron and inverse Compton losses incurred by the electrons radiating at $4.87 \mathrm{GHz}$, using equations given by van der Laan \& Perola (1969).

(20) The position angle, PA, of the line joining the hotspot to the central component of the quasar.

(21) The polarization position angle of the observed peak polarized intensity of the hotspot.

(22) The rotation measure, RM, of the quasar obtained from Simard-Normandin, Kronberg \& Button (1981). Using their measurements, for quasars for which no values of RM are available, a value of the foreground RM has been estimated by averaging the RMs of other extragalactic radio sources located within a radius of $10^{\circ}$ of the quasar. Such values are marked by an asterisk.

(23) The intrinsic position angle of the peak polarization vector of the hotspot, after correcting the values given in column 21 for the Faraday rotation corresponding to the measured or estimated RM.

(24) The difference of the intrinsic position angle of the polarization vector of the hotspot and the line joining the hotspot to the central component (column 23-column 20).

\section{Discussion}

\subsection{THE STRUCTURE OF RADIO LOBES}

As seen in Fig. 1, prominent hotspots are located near the outer edges of the radio lobes of many steep-spectrum quasars in our sample. In some cases, multiple hotspots are found 
within the same radio lobe. Also, in some of the sources, hotspots are found to occur prominently in just one lobe. Although the spatial resolution attained can be expected to influence the observed shape of fine structure in radio lobes, the present observations do reveal several examples of sources where the two radio lobes have structures showing an inversion symmetry about the nucleus. A precession of the energy beams emanating from the nucleus might give rise to such inversion-symmetric structure, the broader portions of which could be the remnants of older hotspots (see Miley 1976; Bridle et al. 1976; Ekers et al. 1978). According to Laing (1983) on the other hand, even such broader components are probably being fed currently with energetic particles continuously flowing out of the compact hotspots, the latter marking the regions where the beam of particles emanating from the nucleus impinge on the intergalactic medium. By a parallel argument, the less compact hotspots of size $\sim 10 \mathrm{kpc}$ seen in some sources might result from beams having relatively large openingangle and thus need not necessarily be much older than compact hotspots existing in the same source (Laing 1983). The present observations do not show any obvious inconsistency with these morphological aspects.

We shall now examine if, for our sample of quasars, the compactness parameter, polarization and size of the hotspots (Section 3.4) vary appreciably as a function of radio luminosity, LAS or redshift of the quasar. It should first be noted that the angular resolution of about 0.4 arcsec attained in the present observations corresponds to a linear resolution of $2.1,3.2$, 4.4 and $5.2 \mathrm{kpc}$ at $z=0.25,0.5,1$ and 2 respectively. To reduce this disparity we have smoothed the maps to a resolution of $0.8 \mathrm{arcsec}$ which corresponds to a linear resolution of $4.2 \mathrm{kpc}$ at $z=0.25$, close to that provided by the full-resolution beam of $0.4 \mathrm{arcsec}$ for sources $z \sim 0.9$. However, this degrading of angular resolution for sources at smaller redshifts did not appreciably alter the value of the compactness parameter $C_{\mathrm{h}}$ taken over all the hotspots given in a radio lobe (Table 2, column 9). Therefore, for simplicity, we shall only consider the parameters given in Tables 1 and 2, which are mostly derived from the full-resolution maps except for the cases when only lower-resolution data are given in Table 1. In order to examine any trends of the types mentioned above, we have divided the quasars broadly into three categories depending upon the size and compactness parameter of their hotspots:

(1) High-brightness quasars (type $\mathrm{H}$ ): Quasars falling in this category have at least one compact, prominent hotspot with $l_{2}<4 \mathrm{kpc}$ and $C_{\mathrm{h}} \geqslant 0.2$. For such quasars, Fig.2(a) displays the distribution of $\triangle \mathrm{PA}$ (Table 2, column 23), the difference between the position angles of the intrinsic linear polarization vector and the line joining the hotspot to the quasar nucleus. It is seen that, for nearly two-thirds of such hotspots, the intrinsic direction of magnetic field lies within $\pm 30^{\circ}$ of the perpendicular to the axis joining the hotspot to the nucleus. This supports the idea that such prominent hotspots are formed as a result of compression of magnetic field at the point where the energy beam from the nucleus impinges on the intergalactic medium (see Laing 1980). The degree of polarization appears to be generally high for hotspots of this type.

(2) Medium-brightness quasars (type $\mathrm{M}$ ): The hotspots in this category of quasars are defined to have $4 \leqslant l_{2} \leqslant 10 \mathrm{kpc}$ and $C_{\mathrm{h}} \geqslant 0.1$. We have also included in this category a few quasar lobes $(0229+34 \mathrm{NW} 2 ; 0901+28 \mathrm{SW} ; 1545+21 \mathrm{NE} 1-\mathrm{NE} 2,1622+23 \mathrm{SW} 2 ; 2131+17$ SW2) containing a hotspot which is compact $\left(l_{2}<4 \mathrm{kpc}\right)$ but not very prominent $(0.1<$ $\left.C_{\mathrm{h}}<0.2\right)$. As seen from Fig. 2(b), the values of $\Delta \mathrm{PA}$ for the medium-brightness quasars are more or less uniformly distributed between $0^{\circ}$ and $90^{\circ}$, in contrast to the peaked distribution found for the high-brightness quasars. In some of the well-resolved hotspots, the magnetic field is found to be circumferential $(1100+77 \mathrm{NW} ; 1545+21 \mathrm{NW} ; 1622+23 \mathrm{NE}$; $1704+60$ NE1). 


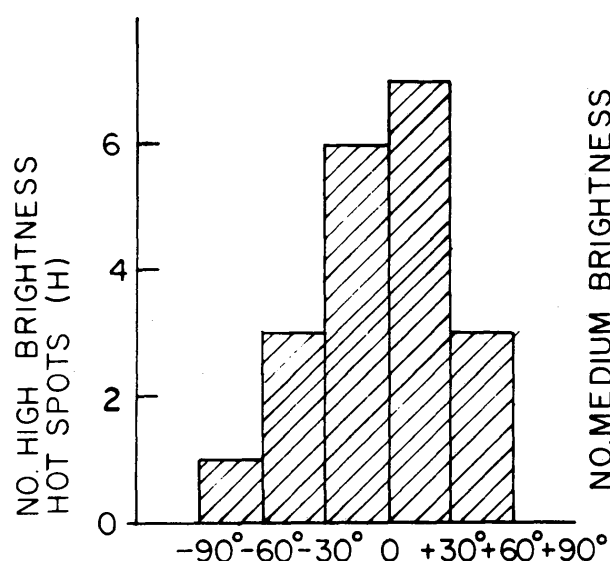

(a)

$\triangle P A$

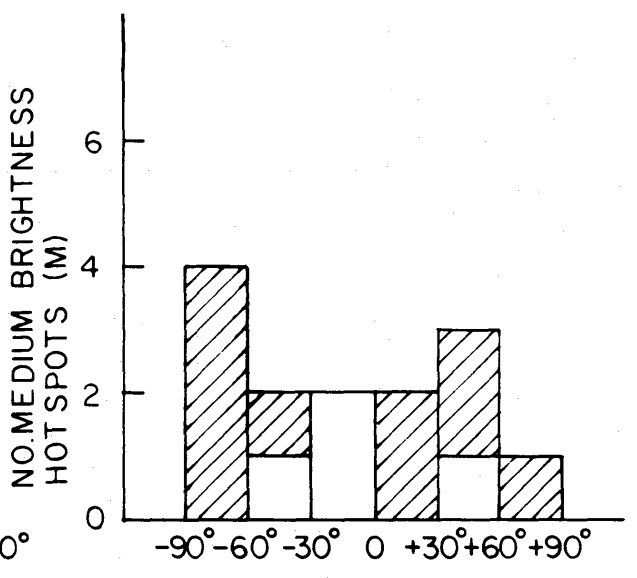

(b)

$\triangle \mathrm{PA}$

Figure 2. The distribution of $\triangle \mathrm{PA}$ giving the difference between the position angles of the intrinsic linear polarization vector and the line joining the hotspot to the quasar nucleus: (a) for type $H$ (high brightness) hotspots and (b) for type $M$ (medium brightness) hotspots. The hotspots with clearly seen circumferential magnetic fields are shown by open boxes.

(3) Low-brightness quasars (type L): In these quasars, no prominent hotspots of size $<10 \mathrm{kpc}$ and $C_{\mathrm{h}}>0.1$ are seen. The radio lobes of this category may be considered to have a relaxed morphology. The absence of prominent hotspots may imply that the extended regions are not now being supplied with energy.

\subsection{THE DEPENDENCE OF HOTSPOT SIZE ON $z, P_{178}$ AND LAS}

Although the measured size can depend weakly on angular resolution, any marked variation of angular sizes of hotspots with $z, P_{178}$ or LAS should still be discernible in our data. In Fig. 3 the angular sizes $\psi_{2}$ of hotspots belonging to the high- and medium-brightness categories (indicated by crosses and dots respectively) are plotted against redshift. The circled points refer to upper limits. Points representing different hotspots in the same quasar are joined by a vertical line. The angular sizes plotted are those measured along the minor axes of the hotspots (Table 1, column 15), as these may be less subject to projection effects. The letters A and B in Fig. 3 refer to 3C 280.1 and 3C9 which show intense extended jets containing a few knots; the angular sizes of the most prominent knots have also been plotted in these cases.

The plotted data points have been compared with the expected variation of angular size with redshift for standard rods having projected lengths of 2.5 and $5 \mathrm{kpc}$ (assuming $q_{0}=0$ ). These are shown in Fig. 3 as full and broken curves respectively. At first glance, the observed sizes of hotspots at high $z$ appear to be somewhat larger than expected for a standard rod. This seems, however, more likely to be an artefact of the degradation of linear resolution with increasing redshift, which might tend to blend multiple hotspots into a single feature. It seems that the size of the hotspots is independent of redshift (Duffett-Smith \& Purvis 1982). Our observations also indicate that sizes of hotspots do not depend on LAS.

In Fig. 4 we have plotted the average physical size $l_{3}$ of hotspots (Table 2, column 13) versus $P_{178}$ of the observed quasars. The symbols are as for Fig. 3. Apparently, the value of $l_{3}$ shows no marked increase with $P_{178}$, nor is $l_{2}$ dependent on $P_{178}$. This is in contrast to the inference made by Readhead \& Hewish (1976) from their interplanetary scintillation (IPS) observations at $80 \mathrm{MHz}$. It is likely that the IPS sizes are affected by the increasing 


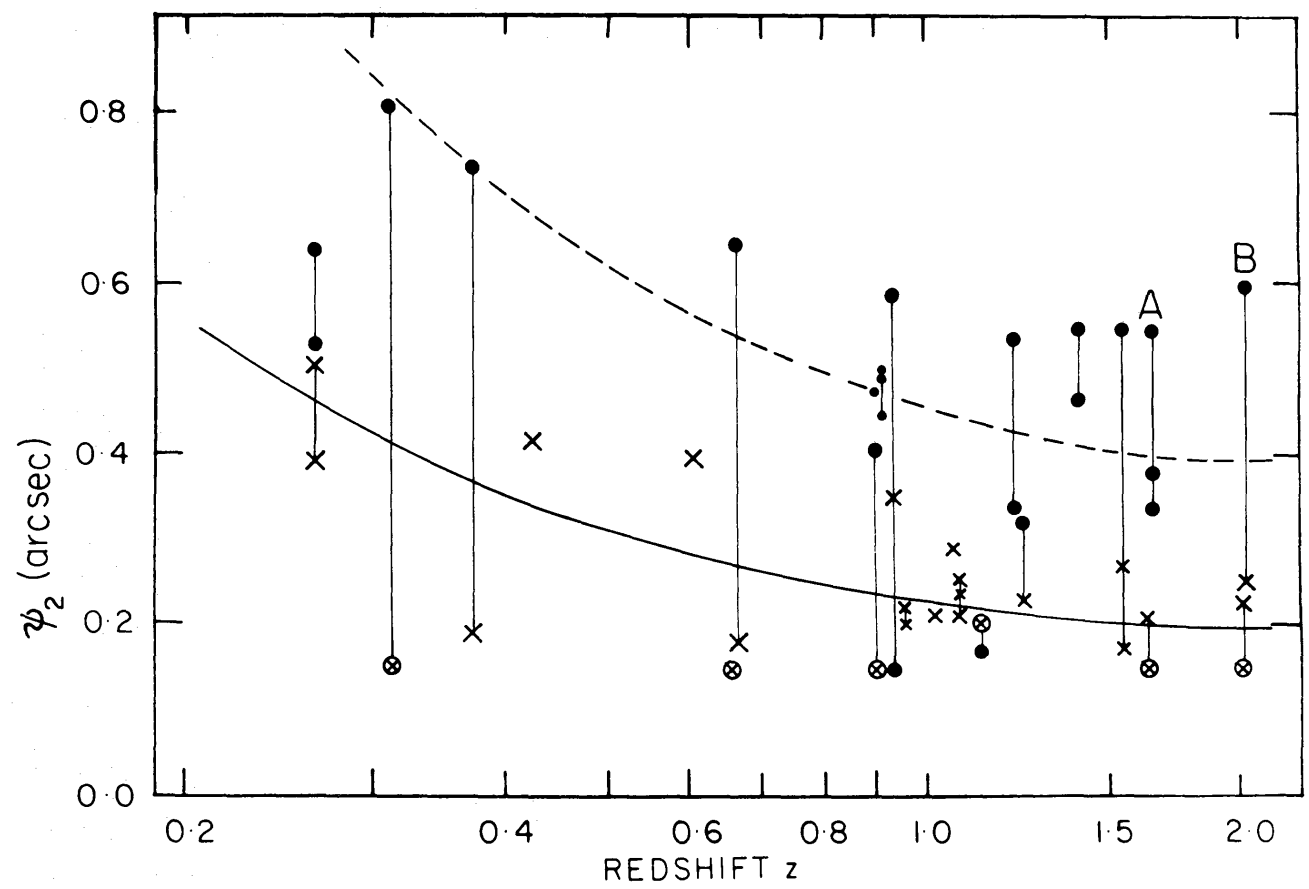

Figure 3. Plot of angular size, $\psi_{2}$, of hotspots versus redshift $z$. Hotspots of type $\mathrm{H}$ and $\mathrm{M}$ are indicated by crosses and dots respectively. The circled values refer to upper limits. Values for the same quasar are joined by a vertical line. The full and broken curves are expected variations for linear sizes of 2.5 and $5 \mathrm{kpc}$ respectively.

blending of multiple hotspots and radio lobes with $z$, owing to the expected degradation of the IPS resolution at higher redshift in terms of linear size (Swarup \& Bhandari 1976; Kapahi 1978; Duffett-Smith \& Purvis 1982). The blending is likely to be much less severe for the present observations which have been made with higher resolution. It is also likely that compact hotspots seen in quasars of high radio luminosity are self-absorbed at $80 \mathrm{MHz}$

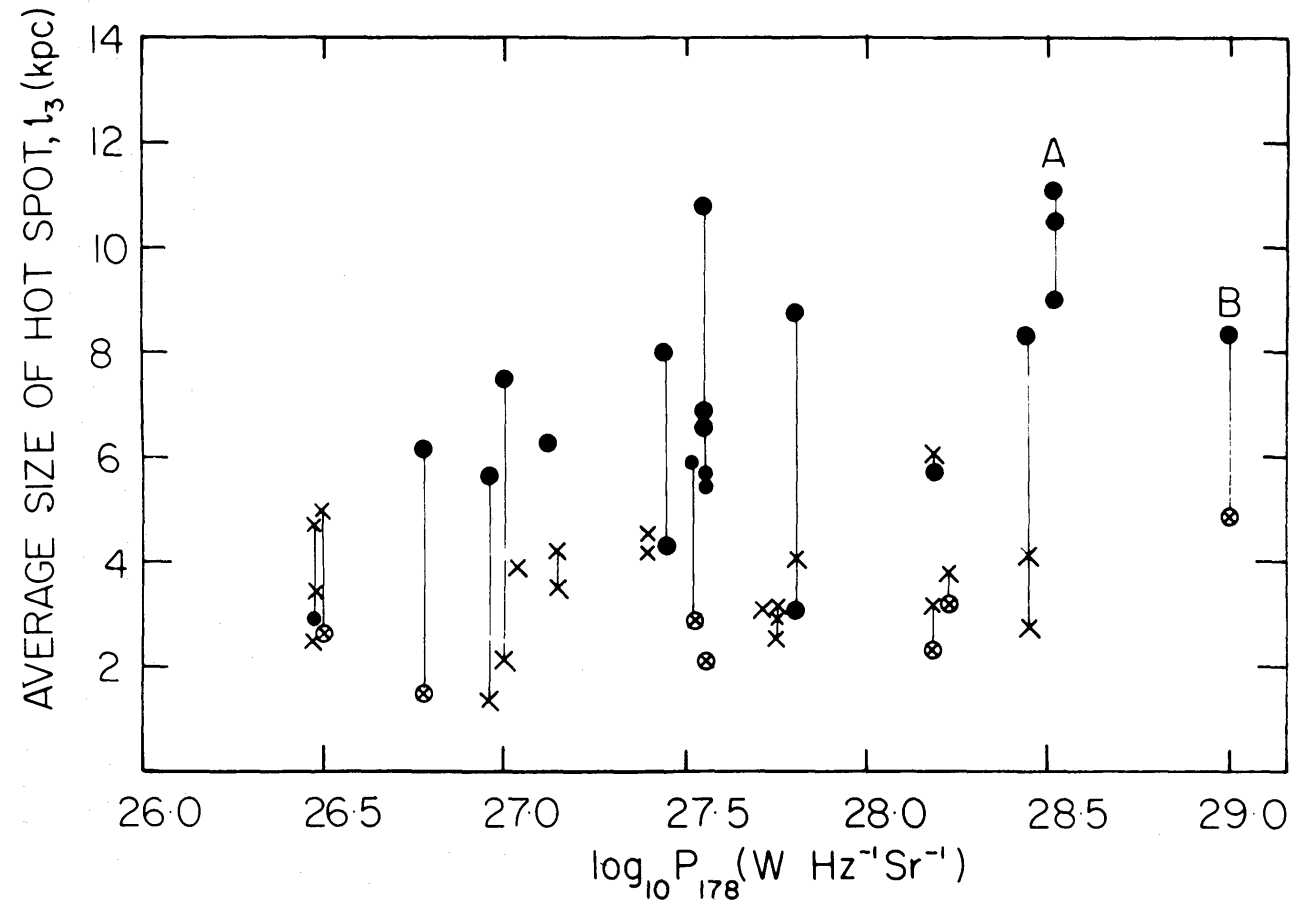

Figure 4. Plot of $l_{3}$ versus $\log _{10} P_{178}$. The symbols are as in Fig. 3. 


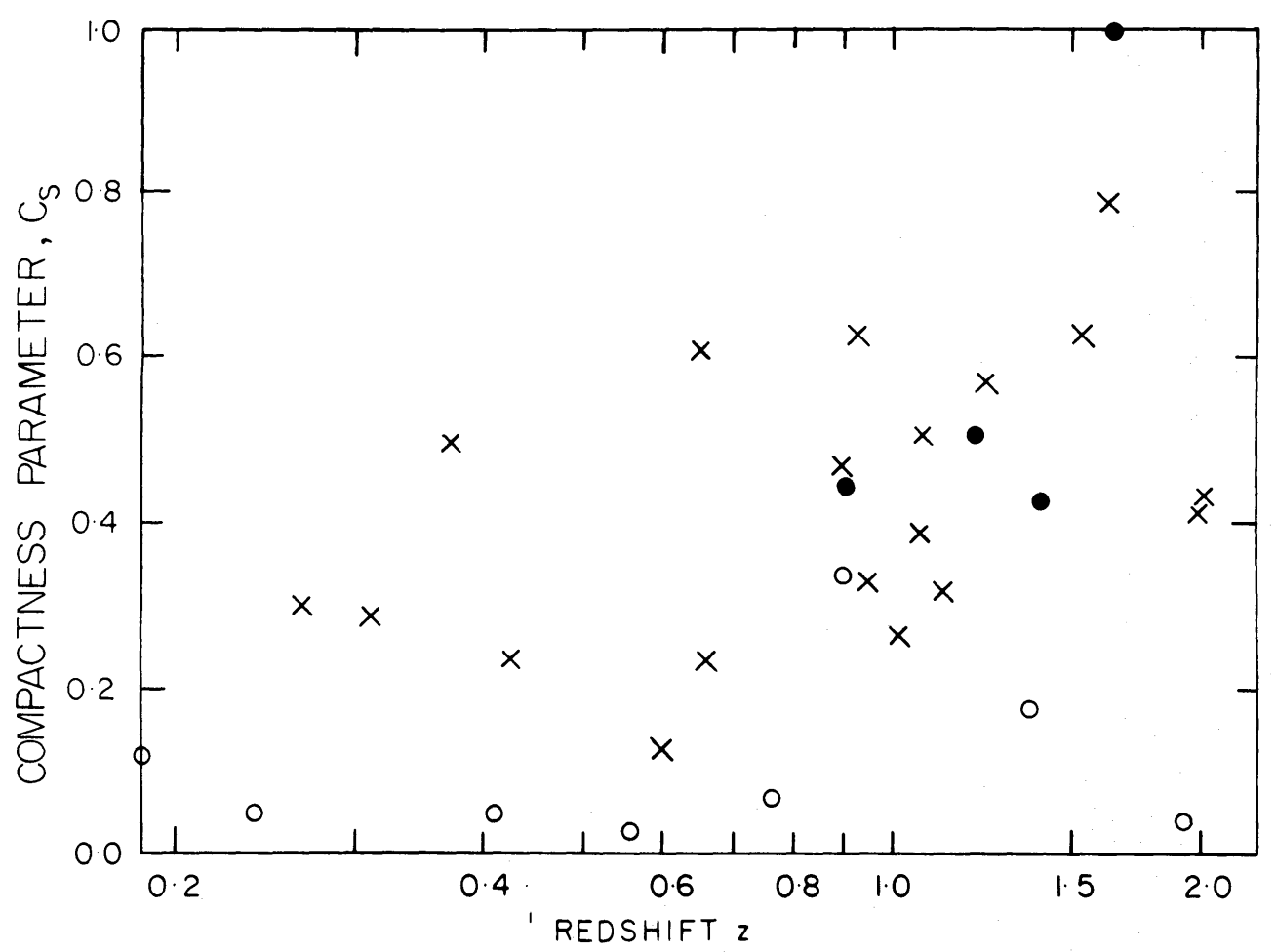

(a)

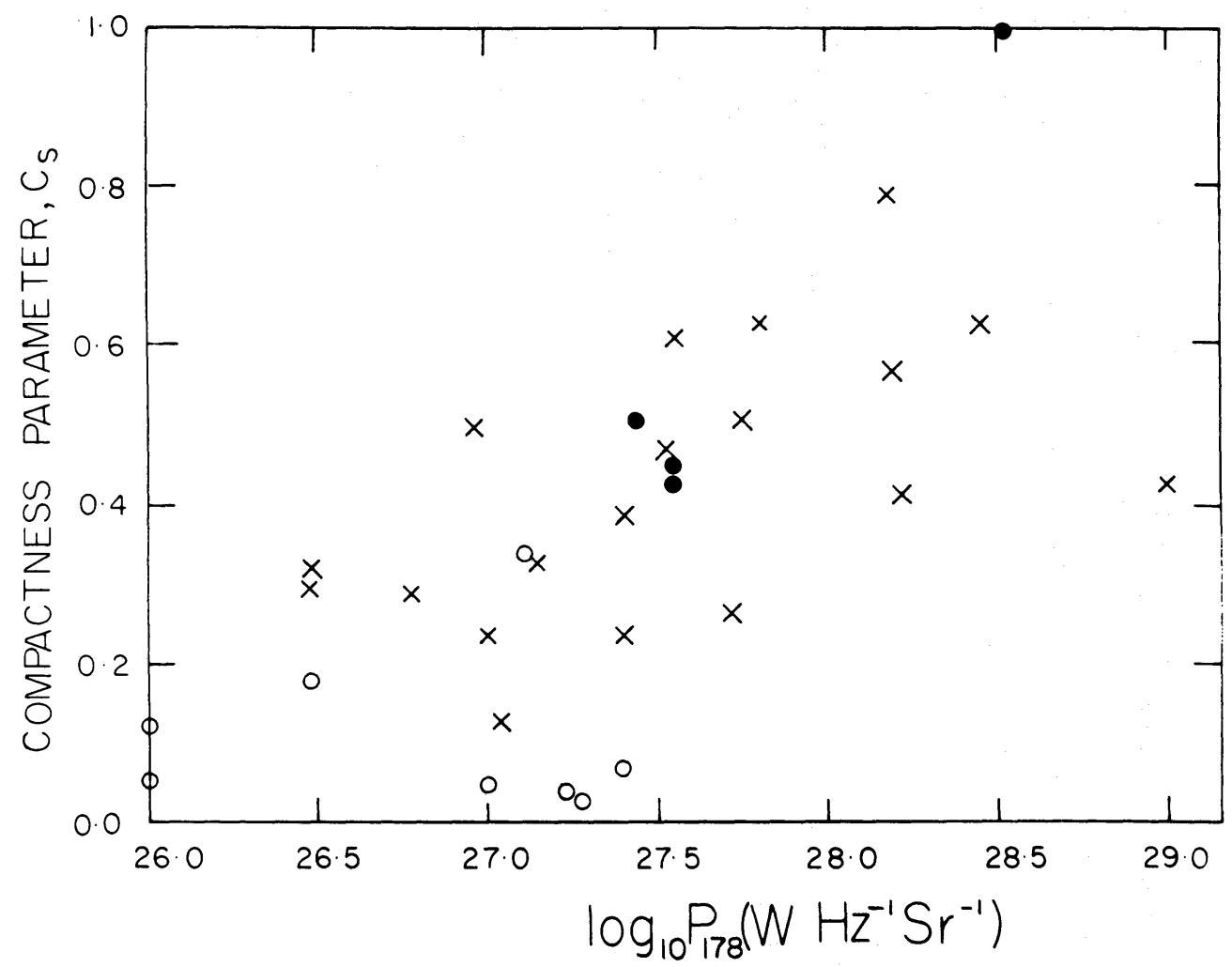

(b)

Figure 5. The compactness parameter $C_{\mathrm{S}}$ is plotted against $z$ in (a) and $\log _{10} \mathrm{P}_{178}$ in (b). The crosses, dots and open circles refer to hotspots of type H, M and L (low brightness) respectively. 
(Laing 1981), which could lead to a positive correlation of $l_{2}$ with $P_{178}$ as found by Readhead \& Hewish (1976).

\subsection{DEPENDENCE OF $C_{\mathrm{s}}$ ON $P_{178}, z$ AND PhySiCAl Size $L$}

From Fig. 5(a, b), the value of the compactness parameter for the entire source, $C_{\mathrm{s}}$, appears to increase significantly with $z$ and hence also with $P_{178}$, for a flux-density limited sample. Actually, $C_{\mathrm{s}}$ seems to be better correlated with $P_{178}$ than with $z$. The correlation between $C_{\mathrm{s}}$ and $P_{178}$ is in accordance with the findings of Jenkins \& McEllin (1977).

Macklin (1982) has noted a trend for the compactness parameter to decrease with the overall physical size $L$ for $993 \mathrm{CR}$ double radio sources, but we find no significant dependence of $C_{\mathrm{s}}$ on $L$ for our sample of 31 quasars located near the upper envelope to the LAS $-z$ diagram.

\subsection{THE RELATION BETWEEN LAS AND $z$}

Fig. 6 is a plot of LAS versus $z$ for 199 quasars selected from 3CR, 4C and B2 samples (Hooley et al. 1978; Stannard \& Neal 1977; Wills 1979; Fanti et al. 1975, 1977). The circled numbers near the bottom edge of the diagram refer to the quasars with LAS $<7$ arcsec falling in the various ranges of redshift described in Section 2. The quasars for which VLA maps were not available are shown by small circles, while those mapped with the VLA are shown by circles, triangles and squares depending upon their radio luminosities falling in the ranges $<10,10-100$ and $>100 \times 10^{26} \mathrm{~W} \mathrm{~Hz}^{-1} \mathrm{sr}^{-1}$, respectively. The indicators are shown filled, half-filled and unfilled for quasars belonging to the high-, medium- and lowbrightness categories respectively. Note that VLA data are available for a total of 34 quasars, 29 being taken from the present sample (see Section 3) and five from other sources (Swarup, Sinha \& Saikia 1982a; Stocke, Christiansen \& Burns 1982; Neff 1982). The new data have significantly improved the information for sources near the upper envelope to the LAS $-z$ diagram.

The expected variation of LAS with $z$ for a quasar of $L=600 \mathrm{kpc}$ in a Friedmann universe with $q_{0}=0$ is shown by a continuous curve in Fig. 6 . The broken line gives the relation LAS $\propto z^{-1}$ expected for a quasar having $L=1000 \mathrm{kpc}$ in a Euclidean universe. These two curves are consistent with the upper envelope of the plotted data points at lower $z$, although at redshifts beyond $\sim 1.5$ a deficiency of large quasars is found relative to the prediction for the Friedmann universe. The deficiency could, however, be merely an artefact of a possible anti-correlation between $P_{178}$ and $L$ (e.g. Jackson 1973; Richter 1973; Stannard \& Neal 1977; Hooley et al. 1978; Masson 1980; Macklin 1982).

It may be expected that radio sources located near the upper envelope to the LAS $-z$ diagram at any $z$ are in a comparatively advanced stage of evolution, in which case the energy supply from the nucleus may decrease and prominent hotspots may not be present in many of these sources. Although we do not find prominent hotspots (type L) for nine out of the 34 quasars for which the VLA data are plotted in Fig. 6, the quasars of lowbrightness category (type L) occur not only near the upper envelope but also at smaller values of LAS. To investigate whether there is an inverse correlation between $L$ and $P$ or a linear-size evolution with $z$ (Wardle \& Miley 1974; Kapahi 1975), it would be desirable

to make high-resolution observations of a larger sample of high-redshift quasars whose flux densities are well below those of the $4 \mathrm{C}$ sample, so that the radio luminosities of these objects are comparable with those of low-redshift quasars. 


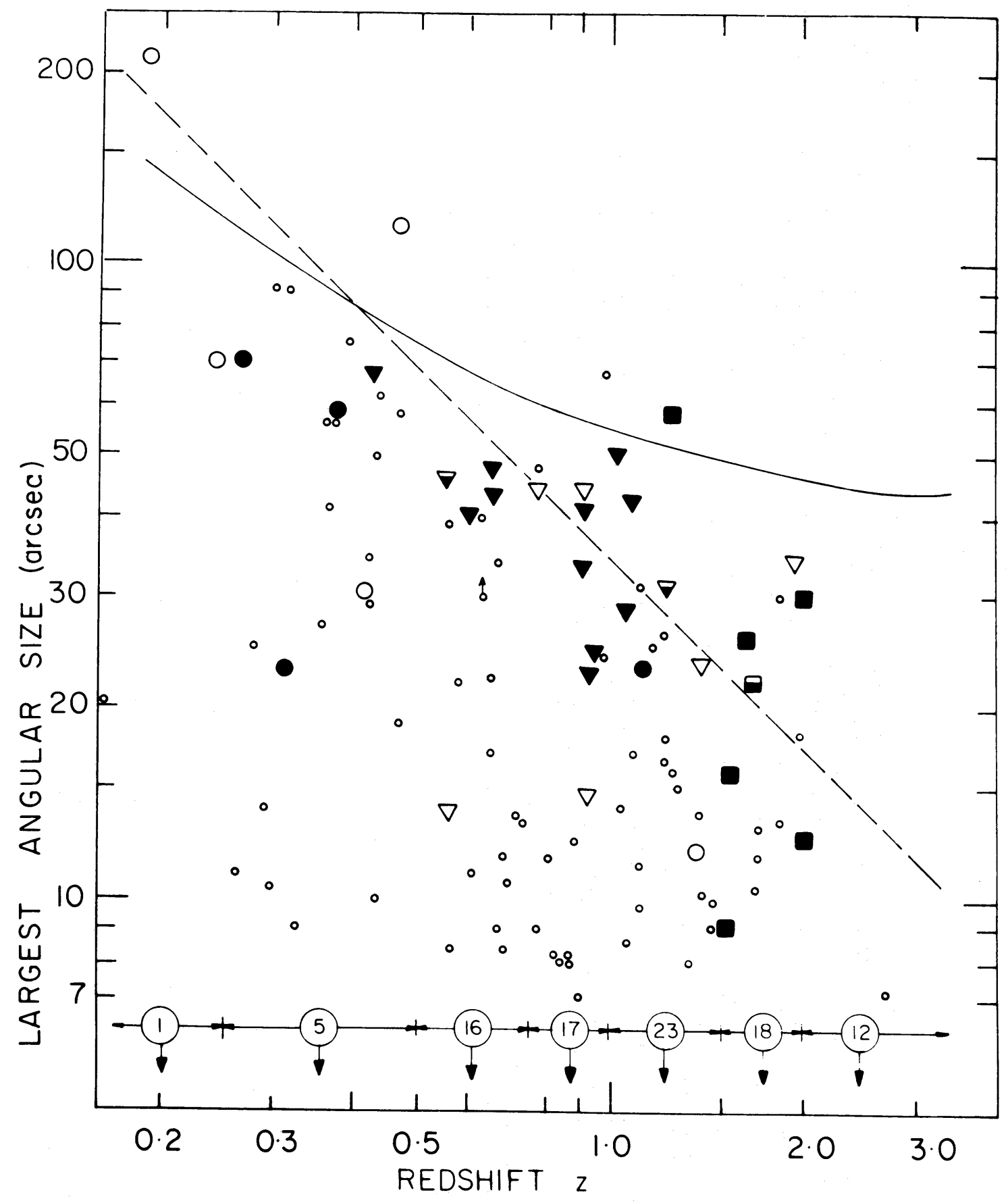

Figure 6. Plot of Largest Angular Size against $z$. Quasars with $\log _{10} P_{178}<10,10-100$ and $>100 \times 10^{26}$ $\mathrm{W} \mathrm{Hz}^{-1} \mathrm{sr}^{-1}$ are shown with circles, triangles and squares respectively. The symbols are filled, half-filled and open for type H, M and L hotspots. Quasars for which no VLA data are available are marked with small circles.

\subsection{THE CENTRAL COMPONENTS}

In several quasars of the present sample, the central component is partially resolved, indicating a size of $1-2 \mathrm{kpc}$. In fact the general trend is that the ratio of the peak to integrated flux density of the central components, $f_{\mathfrak{n}}$, is significantly less than 1 , the average value being 0.87 for 25 quasars with central components (Table 2, see column 10 against the row $\mathrm{CN})$. The same value of $f_{\mathrm{n}}$ is found for sources having the self-calibrated maps and hence 
this estimate is unlikely to be a consequence of any atmospheric phase-deviations arising across the aperture of the VLA during out observations. It appears therefore that the extended emission accounts for nearly 10 per cent of the total radio output from the central components at $5 \mathrm{GHz}$, on average. This estimate is unlikely to have been much affected by any Doppler boosting of the nuclear emission due to relativistic motions, as the overall angular sizes of the sources in the present sample are among the largest known at any given redshift and hence their radio axes are expected to lie well away from the line of sight.

The fraction, $f_{c}$, of the flux density of the central component to the total flux density of the 31 quasars (Table 2, column 10) is found to have a median value of $\sim 0.1$ at $5 \mathrm{GHz}$. The median value of $f_{\mathrm{c}}$ for 23 quasars with $f_{\mathrm{c}}<0.25$ is 0.08 . There seems a trend for $f_{\mathrm{c}}$ to increase with redshift, as seven out of the eight quasars having $f_{\mathrm{c}}>0.25$ lie at $z \gtrsim 0.9$. The higher $f_{\mathbf{c}}$ for high-redshift quasars may be a consequence of relativistic beaming if their axes lie closer to the line of sight (Ulvestad et al. 1981; Orr \& Browne 1982). For quasars with higher $f_{\mathfrak{c}}$, Kapahi \& Saikia (1984) have shown that the degree of misalignment of the outer lobes with respect to the central core is, on average, higher. A similar trend is noticed for some quasars with higher $f_{\mathrm{c}}$ in our sample $(0957+00 ; 1244+32 ; 1602+00)$ as may be seen from Fig. 1 and Table 2.

From high-resolution observations of $323 \mathrm{CR}$ quasars, Orr \& Browne (1982) have estimated the ratio $R_{\mathrm{T}}$ of the flux density of the beamed central component $(\alpha=0)$ to that of the unbeamed extended components $(\alpha=-1)$ when the radio axis is transverse to the line of sight. They have estimated that $R_{\mathrm{T}}=0.024$ at $5 \mathrm{GHz}$ (emitted) and the Lorentz factor $\gamma=5$. Assuming that the 23 quasars in our sample located near the upper envelope to the LAS $-z$ diagram, and having $f_{\mathrm{c}}<0.25$, subtend an angle to the line of sight uniformly distributed between $60^{\circ}$ and $90^{\circ}$, the average Doppler-enhancement factor is 1.32 for $\gamma=5$. The median value of $z$ for the 23 quasars in 0.8 . Hence, we estimate $R_{\mathrm{T}}(5 \mathrm{GHz}$ emitted) $\cong(1.32)^{-1} \bar{f}_{\mathrm{c}} \bar{f}_{\mathrm{n}}\left(1-\bar{f}_{\mathrm{c}} \bar{f}_{\mathrm{n}}\right)^{-1}(1+\mathrm{z})^{-1} \cong 0.033$, taking the averages $\bar{f}_{\mathrm{c}}=0.08$ and $\bar{f}_{\mathrm{n}}=0.9$ according to our observations at $5 \mathrm{GHz}$. This estimate is comparable to, though on the higher side of, the value of $R_{\mathrm{T}}=0.024$ estimated by Orr \& Brown (1982).

\section{Conclusions}

High-resolution observations have been made of 28 quasars selected from the $3 \mathrm{C}$ and $4 \mathrm{C}$ complete samples and three from the B2 sample of quasars. It is found that:

(1) About a quarter of the quasars located near the upper envelope to the LAS $-z$ diagram show only relaxed lobes in which no prominent hotspots are seen. This may imply that their radio lobes are currently not being supplied with energy.

(2) About 60 per cent of the quasars contain at least one compact hotspot of size $<4 \mathrm{kpc}$ in the direction of minor axis and having a compactness parameter $C_{\mathrm{h}}>0.2$. For nearly twothirds of these hotspots, the magnetic field directions are found to be nearly perpendicular to the lines joining the hotspots to the quasar nuclei, supporting the hypothesis that a prominent hotspot is formed as a result of compression of magnetic field at the point where the energy beam from the nucleus impinges on the intergalactic medium.

(3) The linear sizes of hotspots show no marked dependence on $z$, LAS or $P_{178}$, but the fraction of flux density in the hotspots to that of the radio lobes, $C_{\mathrm{s}}$, is well correlated with $P_{178}$, confirming the trend noticed by Jenkins \& McEllin (1977).

(4) There seems to be a deficiency of large quasars relative to the prediction of a Friedmann universe, for $z \gtrsim 1.5$. This may arise either from an inverse correlation between $L$ and $\mathrm{P}$, or from the evolution of linear size with $z$. High-resolution observations for a sample 
of high-redshift quasars, complete to much lower flux density level than that of the $4 \mathrm{C}$ sample, may allow a choice between these possibilities.

\section{Acknowledgments}

We are grateful to Gopal-Krishna, V. K. Kapahi, P. P. Kronberg and R. A. Laing for valuable comments; D. J. Saikia for providing the data of $1739+18$ and for self-calibration of four of the sources described here; C. J. Salter for help during the observations and valuable comments; P. Katgert for advice during data reduction made at Leiden; and R. Nanjan, A. K. Singal and D. G. Banhatti for assistance in data reduction. GS is grateful to the Bosscha Foundation for travel support for the VLA observations and to the University of Leiden for a six-month Visiting Professorship.

\section{References}

Argue, A. N. \& Kenworthy, C. M., 1972. Mon. Not. R. astr. Soc., 160, 197.

Baars, J. W. M., Genzel, R., Pauliny-Toth, I. I. K. \& Witzel, A., 19.77. Astr. Astrophys., 61, 99.

Baldwin, J. A., 1975. Astrophys. J., 201, 26.

Bridle, A. H., Davis, M. M., Meloy, D. A., Fomalont, E. B., Strom, R. G. \& Wills, A. G., 1976. Nature, 262, 179.

Cotton, W. D., Owen, F. N. \& Ghigo, F. D., 1975. Astr. J., 80, 353.

Duffett-Smith, P. J. \& Purvis, A., 1982. Extragalactic Radio Sources, IAU Symp. 97, p. 59, eds Heeschen, D. S. \& Wade, C. M., Reidel, Dordrecht, Holland.

Ekers, R. D., Fanti, R., Lari, C. \& Parma, P., 1978. Nature, 276, 588.

Fanti, C., Fanti, R., Ficarra, A., Formiggini, L., Giovannini, G., Lari, C. \& Padrielli, L., 1975. Astr. Astrophys. Suppl., 19, 143.

Fanti, C., Fanti, R., Formiggini, L., Lari, C. \& Padrielli, L., 1977. Astr. Astrophys. Suppl., 28, 351.

Fomalont, E. B., 1981. Origin of Cosmic Rays, IAU Symp. 94, p. 111, eds Setti, G., Spada, G. \& Wolfendale, A. W., Reidel, Dordrecht, Holland.

Ghigo, F. D. \& Owen, F. N., 1973. Astr. J., 78, 848.

Gopal-Krishna \& Swarup, G., 1977. Mon. Not. R. astr. Soc., 178, 265.

Gower, J. F. R., Scott, P. F. \& Wills, D., 1967. Mem. R. astr. Soc. 71, 49.

Hewitt, A. \& Burbidge, G., 1980. Astrophys. J. Suppl., 43, 57.

Högbom, J. A., 1974. Astr. Astrophys. Suppl., 15, 417.

Hooley, A., Longair, M. S. \& Riley, J. M., 1978. Mon. Not. R. astr. Soc., 182, 127.

Jackson, J. C., 1973. Mon. Not. R. astr. Soc., 162, 11P.

Jenkins, C. J. \& McEllin, M., 1977. Mon. Not. R. astr. Soc., 180, 219.

Jenkins, C. J., Pooley, G. G. \& Riley, J. M., 1977. Mem. R. astr. Soc., 84, 61.

Kapahi, V. K., 1975. Mon. Not. R. astr. Soc., 172, 513.

Kapahi, V. K., 1978. Astr. Astrophys., 67, 157.

Kapahi, V. K., 1981.J. Astrophys. Astr., 2, 43.

Kapahi, V. K., Gopal-Krishna \& Joshi, M. N., 1974. Mon. Not. R. astr. Soc., 167, 299.

Kapahi, V. K. \& Saikia, D. J., 1984.J. Astrophys. Astr., in press.

Kapahi, V. K. \& Schilizzi, R. T., 1979. Nature, 277, 610.

Kronberg, P. P., Clarke, J. N. \& van den Bergh, S., 1980. Astr. J., 85, 973.

Kühr, H., Nauber, U., Pauliny-Toth, I. I. K. \& Witzel, A., 1979. A Catalogue of Radio Sources, MaxPlanck Institute for Radio Astronomy, Bonn.

Laing, R. A., 1980. Mon. Not. R. astr. Soc., 193, 439.

Laing, R. A., 1981. Mon. Not. R. astr. Soc., 195, 261.

Laing, R. A., 1983. Winter school on Extragalactic Energetic Sources, Tata Institute of Fundamental Research, Bangalore, India.

Lonsdale, C. J. \& Morison, I., 1983. Mon. Not. R. astr. Soc., 203, 833.

McEwan, N. J., Browne, I. W. A. \& Crowther, J. H., 1975. Mem. R. astr. Soc., 80, 1.

Mackay, C. D., 1969. Mon. Not. R. astr. Soc., 145, 31.

Macklin, J. T., 1982. Mon. Not. R. astr. Soc., 199, 1119.

Masson, C. R., 1980. Astrophys. J., 242, 8. 
Miley, G. K., 1971. Mon. Not. R. astr. Soc., 152, 477.

Miley, G. K., 1976. Proc. NATO Summer School, Physics of Non-Thermal Sources, p. 1, Reidel, Dordrecht, Holland.

Miley, G. K., 1980. Ann. Rev. Astr. Astrophys., 18, 165.

Miley, G. K. \& Hartsuiker, A. P., 1978. Astr. Astrophys. Suppl., 34, 129.

Neff, S. G., 1982. Extragalactic Radio Sources, IAU Symp. 97, p. 137, eds Heeschen, D. S. \& Wade, C. M., Reidel, Dordrecht, Holland.

Oemler, A., Gunn, J. E. \& Oke, J. B., 1972. Astrophys. J., 176, L47.

Orr, M. J. L. \& Browne, I. W. A., 1982. Mon. Not. R. astr. Soc., 200, 1067.

Pacholczyk, A. G., 1970. Radio Astrophysics, W. H. Freeman, San Francisco.

Pooley, G. G. \& Henbest, S. N., 1974. Mon. Not. R. astr. Soc., 169, 447.

Potash, R. I. \& Wardle, J. F. C., 1979. Astr. Astrophys., 17, 432.

Readhead, A. C. S. \& Hewish, A., 1976. Mon. Not. R. astr. Soc., 176, 571.

Richter, G. M., 1973. Astrophys. Lett., 13, 63.

Riley, J. M. \& Pooley, G. G., 1975. Mem. R. astr. Soc., 80, 105.

Schwab, F. R., 1980. Proc. Soc. Photo-opt. Instrum. Engng, 231, 18.

Simard-Normandin, M., Kronberg, P. P. \& Button, S., 1981. Astrophys. J. Suppl., $45,97$.

Smith, H. E. \& Spinrad, H., 1980. Publs. astr. Soc. Pacif., 92, 553.

Stannard, D. \& Neal, D. S., 1977. Mon. Not. R. astr. Soc., 179, 719.

Stocke, J., Christiansen, W. \& Burns, J. A., 1982. Extragalactic Radio Sources, IAU Symp. 97, p. 39, eds Heeschen, D. S. \& Wade, C. M., Reidel, Dordrecht, Holland.

Swarup, G. \& Bhandari, S. M., 1976. Astrophys. Lett., 17, 31.

Swarup, G., Sinha, R. P. \& Saikia, D. J., 1982a. Mon. Not. R. astr. Soc., $201,393$.

Swarup, G., Sinha, R. P. \& Salter, C. J., 1982b. Extragalactic Radio Sources, IA U Symp. 97, p. 411, eds Heeschen, D. S. \& Wade, C. M., Reidel, Dordrecht, Holland.

Thompson, A. R., Clark, B. G., Wade, C. M. \& Napier, P. J., 1980. Astrophys. J. Suppl., 44, 151.

Ulrich, M. H., 1976. Astrophys. J., 206, 364.

Ulvestad, J. S., Johnston, K., Perley, R. \& Fomalont, E. B., 1981. Astr. J., 86, 1010.

van der Laan, H. \& Perola, G. C., 1969. Astr. Astrophys., 3, 468.

Wall, J. V., 1972. Austr. J. Phys. Astrophys. Suppl., 24, 1.

Wardle, J. F. C. \& Miley, G. K., 1974. Astr. Astrophys., 30, 305.

Wardle, J. F. C. \& Potash, R. I., 1982. Extragalactic Radio Sources, IAU Symp. 97, p. 129, eds Heeschen, D. S. \& Wade, C. M., Reidel, Dordrecht, Holland.

Wills, D., 1979. Astrophys. J. Suppl., 39, 291.

Wills, D. \& Lynds, R., 1978. Astrophys. J. Suppl., 36, 317. 